\title{
Stationary States of the Nonlinear Dirac Equation: A Variational Approach
}

\section{Maria J. Esteban, Eric Séré}

CEREMADE (URA CNRS 749), Université Paris-Dauphine, Place de Lattre de Tassigny, F-75775 Paris Cedex 16, France

Received: 1 August 1993

Abstract: In this paper we prove the existence of stationary solutions of some nonlinear Dirac equations. We do it by using a general variational technique. This enables us to consider nonlinearities which are not necessarily compatible with symmetry reductions.

\section{Section 1. Introduction and Main Results}

The nonlinear Dirac equation has been widely used to build relativistic models of extended particles by means of nonlinear Dirac fields. A general form of this equation in the case of an elementary fermion is

$$
i \gamma^{\mu} \partial_{\mu} \psi-m \psi+\gamma^{0} \nabla F(\psi)=0
$$

Here, $\psi: \mathbb{R}^{4} \rightarrow \mathbb{C}^{4}, \partial_{\mu} \psi=\frac{\partial}{\partial x^{\mu}} \psi, 0 \leqq \mu \leqq 3$, we have used Einstein's convention for summation over $\mu, m$ is a positive constant, $F: \mathbb{C}^{4} \rightarrow \mathbb{R}$ models a nonlinear interaction and $\gamma^{\mu}$ are the $4 \times 4$ Pauli-Dirac matrices:

$$
\gamma^{0}=\left(\begin{array}{cc}
I & 0 \\
0 & -I
\end{array}\right) \text { and } \gamma^{k}=\left(\begin{array}{cc}
0 & \sigma^{k} \\
-\sigma^{k} & 0
\end{array}\right) \text { for } k=1,2,3
$$

with

$$
\sigma^{1}=\left(\begin{array}{ll}
0 & 1 \\
1 & 0
\end{array}\right), \sigma^{2}=\left(\begin{array}{cc}
0 & -i \\
i & 0
\end{array}\right), \sigma^{3}=\left(\begin{array}{cc}
1 & 0 \\
0 & -1
\end{array}\right) .
$$

Note that $x^{0}$ plays here the role of time. Throughout this paper we assume that $F$ satisfies $F \in C^{2}$ and

$$
F\left(e^{i \theta} \psi\right)=F(\psi) \quad \text { for all } \theta
$$

Different functions $F$ have been used to model various types of selfcouplings. For a review on this and historical background see for instance [14]. 
Stationary states of the nonlinear Dirac equation are considered as particle-like solutions. These solutions are in some sense solitons which propagate without changing their shape.

Stationary solutions are functions of the type

$$
\psi\left(x^{0}, x\right)=e^{-i \omega x^{0}} \varphi(x),
$$

where by $x$ we denote $\left(x^{1}, x^{2}, x^{3}\right) \in \mathbb{R}^{3}$, and such that $\varphi$ is a non-zero localized solution of the following stationary nonlinear Dirac equation:

$$
i \gamma^{k} \partial_{k} \varphi-m \varphi+\omega \gamma^{0} \varphi+\gamma^{0} \nabla F(\varphi)=0 \text { in } \mathbb{R}^{3} .
$$

Here, we have used the repeated index convention for summation over the three values of $k$. We will do this throughout the paper.

By "localized," we mean that $\varphi \in W^{1, q}\left(\mathbb{R}^{3}, \mathbb{C}^{4}\right)$ for any $2 \leqq q<\infty$ and $F(\varphi) \in L^{1}\left(\mathbb{R}^{3}, \mathbb{R}\right)$.

The above equation has a variational structure. Indeed it is the Euler-Lagrange equation corresponding to the following functional:

$$
I^{\omega}(\varphi)=\int_{\mathbb{R}^{3}}\left(-\frac{1}{2}\left(i \gamma^{0} \gamma^{k} \partial_{k} \varphi, \varphi\right)+\frac{m}{2} \bar{\varphi} \varphi-\frac{\omega}{2}|\varphi|^{2}-F(\varphi)\right) d x,
$$

where $($,$) is the usual scalar product in \mathbb{C}^{4}$ and $\bar{\varphi} \varphi$ denotes $\left(\gamma^{0} \varphi, \varphi\right)$.

Existence of solutions of (1.5), i.e. of critical points of $I^{\omega}$ has been proved in $[5,6,9$ and 13] in the particular case when

$$
F(\varphi)=\frac{1}{2} G(\bar{\varphi} \varphi), \quad G \in C^{2}(\mathbb{R}, \mathbb{R}), \quad G(0)=0
$$

under suitable conditions on $G$, with $\omega \in(0, m)$. When $(1.7)$ holds, one can use a particular ansatz for the solutions:

$$
\varphi(x)=\left(\begin{array}{c}
v(r)\left(\begin{array}{l}
1 \\
0
\end{array}\right) \\
i u(r)\left(\begin{array}{c}
\cos \theta \\
\sin \theta
\end{array} e^{i \phi}\right.
\end{array}\right)
$$

and then Eq. (1.5) reduces to the O.D.E. system

$$
\left\{\begin{aligned}
u^{\prime}+\frac{2 u}{r} & =v\left[g\left(v^{2}-u^{2}\right)-(m-\omega)\right] \\
v^{\prime} & =u\left[g\left(v^{2}-u^{2}\right)-(m+\omega)\right]
\end{aligned}\right.
$$

where $g(s)=G^{\prime}(s)$.

In the above papers the system (1.9) is solved by a shooting method which yields an infinity of localized solutions for (1.9). We will state the precise results of $[5,9$ and 13] in Sect. 4.

The particular form (1.7) corresponds to the so-called Soler model, and has been widely studied (see $[14,17]$ ). There are other models of selfcoupling for which the ansatz (1.8) is no more valid, for instance the following nonlinearity (see $[10,14]$ ):

$$
F(\varphi)=\frac{1}{2}|\bar{\varphi} \varphi|^{2}+b\left|\bar{\varphi} \gamma^{5} \varphi\right|^{2}
$$

with $b \neq 0, \bar{\varphi}=\gamma^{0} \varphi$ and $\gamma^{5}=\gamma^{0} \gamma^{1} \gamma^{2} \gamma^{3}$. 
In this paper we prove, by a variational method, the existence of non-zero critical points of $I^{\omega}$, and therefore of localized solutions of (1.5), both in the case when $F$ satisfies (1.7) and in the general case.

When $F$ satisfies (1.7), we actually solve the O.D.E. system (1.9) by a variational method to obtain the existence of an infinity of solutions of (1.9) under assumptions slightly less general than those in $[5,9,13]$. The main assumption we have to add is that $G$ satisfies

$$
G^{\prime}(x) \cdot x \geqq \theta G(x), \quad \theta>1, \forall x \in \mathbb{R} .
$$

This is a super-linearity condition on $G$. This kind of technical condition often appears as necessary to use variational techniques when solving nonlinear PDE's.

If $F$ is a general function which does not necessarily satisfy (1.7), we also obtain an existence result for (1.5) but only for functions $F$ which grow more slowly than $|\varphi|^{3}$ at infinity. This limitation comes from the fact that we work in the space $H^{1 / 2}\left(\mathbb{R}^{3}, \mathbb{C}^{4}\right)$. Hence we are not able yet to treat nonlinearities like those satisfying (1.10). Nevertheless we believe that the method used in this paper should be useful to extend our results and treat more general nonlinearities. Our results are concerned, for instance, with nonlinearities as

$$
\frac{1}{2}|\bar{\varphi} \varphi|^{\alpha}+b\left|\bar{\varphi} \gamma^{5} \varphi\right|^{\beta}, \quad 1<\alpha, \beta<\frac{3}{2}, b \geqq 0 .
$$

Let us now state our main results.

Theorem 1. Let $F: \mathbb{C}^{4} \rightarrow \mathbb{R}$ satisfy (1.7) with $G \in C^{2}(\mathbb{R}, \mathbb{R})$. Denoting by $g$ the first derivative of $G$, we make the following assumptions:

$$
\begin{gathered}
g(x) \cdot x \geqq \theta G(x), \quad \theta>1, \forall x \in \mathbb{R} . \\
G(0)=g(0)=0 . \\
G(x) \geqq 0(\forall x \in \mathbb{R}) \text { and } G\left(A_{0}\right)>0 \text { for some } A_{0}>0 . \\
0<\omega<m .
\end{gathered}
$$

Then there is an infinity of solutions of Eq. (1.5) in $\bigcap_{2 \leq q<+\infty} W^{1, q}\left(\mathbb{R}^{3}, \mathbb{C}^{4}\right)$. Each of them is found by a min-max on the functional $I^{\omega}$. They are of the form (1.8), so they correspond to classical solutions of (1.9) on $\mathbb{R}_{+}$, decreasing exponentially at infinity.

For more general nonlinearities $F$, not compatible with the ansatz (1.8), we have

Theorem 2. Let $F(\varphi)=\lambda\left(|\varphi \bar{\varphi}|^{\alpha_{1}}+b\left|\bar{\varphi} \gamma^{5} \varphi\right|^{\beta}\right)$, with $1<\alpha, \beta<\frac{3}{2} ; \lambda, b>0$.

Then there exists a non-zero solution of $(1.5)$ in $\bigcap_{2 \leqq q<+\infty} W^{1, q}\left(\mathbb{R}^{3}, \mathbb{C}^{4}\right)$ for every $\omega \in(0, m)$.

In fact, we will prove a more general result:

Theorem 3. Assume that $F: \mathbb{C}^{4} \rightarrow \mathbb{R}$ satisfies $:$

$$
0 \leqq F(\varphi) \leqq a_{1}\left(|\varphi|^{\alpha_{1}}+|\varphi|^{\alpha_{2}}\right), \quad \forall \varphi \in \mathbb{C}^{4} .
$$


Here, $a_{1}>0$ and $2<\alpha_{1} \leqq \alpha_{2}<3$.

$$
\begin{gathered}
F \in C^{2}\left(\mathbb{C}^{4}, \mathbb{R}\right), \quad F^{\prime}(0)=F^{\prime \prime}(0)=0, \\
\left|F^{\prime \prime}(\varphi)\right| \leqq a_{2}|\varphi|^{\alpha_{2}-2}, \quad a_{2}>0, \quad \text { for }|\varphi| \text { large. } \\
\nabla F(\varphi) \cdot \varphi \geqq \alpha F(\varphi), \quad \alpha>2, \forall \varphi \in \mathbb{C}^{4} . \\
\exists \beta>3, \forall \delta>0, \exists C_{\delta}>0, \forall \varphi \in \mathbb{C}^{4}, \\
|\nabla F(\varphi)| \leqq\left(\delta+C_{\delta} F(\varphi)^{\frac{1}{\beta}}\right)|\varphi| . \\
F(\varphi) \geqq a_{3}|\varphi \bar{\varphi}|^{v}-a_{4}, \quad v>1, a_{3}, a_{4}>0, \forall \varphi \in \mathbb{C}^{4} .
\end{gathered}
$$

Then, for every $\omega \in(0, m)$, we can find a non-zero solution of (1.5) in $\bigcap_{2 \leqq q<+\infty} W^{1, q}\left(\mathbb{R}^{3}, \mathbb{C}^{4}\right)$.

Theorem 2 is clearly a consequence of Theorem 3 .

To prove Theorems 1 to 3 we will use a linking argument. For a description of the general linking method see [7]. In the case of Theorems 2,3, we need some ideas introduced in $[11,19]$ in the context of Hamiltonian systems, and we use the concentration-compactness theory [12].

This paper is organized as follows. The second section is devoted to the proof of the existence of linking critical levels for the functional $I^{\omega}$. In Sect. 3 we prove some auxiliary results and a priori estimates. Theorem 1 is proved in Sect. 4 and Theorem 2 and 3, in Sect. 5.

\section{Section 2. The Linking Argument}

We consider a functional of the form

$$
I^{\omega}(\varphi)=I^{0}(\varphi)-\frac{\omega}{2}\|\varphi\|_{L^{2}\left(\mathbb{R}^{3}, \mathbb{C}^{4}\right)}^{2}, \quad \varphi \in H^{1 / 2}\left(\mathbb{R}^{3}, \mathbb{C}^{4}\right),
$$

where

$$
I^{0}(\varphi)=\frac{1}{2} \int_{\mathbb{R}^{3}}\left[\varphi,\left(-i \gamma^{0} \gamma^{k} \partial_{k}+m \gamma^{0}\right) \varphi\right] d^{3} x-\int_{\mathbb{R}^{3}} F(\varphi) d^{3} x,
$$

under the assumptions (H5), (H6), (H9), and for $0<\omega<m$. $I^{\omega}$ is well defined and of class $C^{2}$ on $E=H^{1 / 2}\left(\mathbb{R}^{3}, \mathbb{C}^{4}\right)$.

We want to prove Theorems 1 and 3 thanks to linking arguments on $I^{\omega}$. For Theorem 1, the linking argument will work in the subspace of $E$ whose elements are of the form (1.8), and will give a nondecreasing sequence $\left(c_{N}\right)_{N \geqq 1}$ of positive min-max levels, assuming $F$ of the form $G(\varphi \bar{\varphi})$. This will be done in part $A$ of this section.

For Theorem 3, the linking will give one positive min-max level $c(\omega)$.

When studying these linkings for $I^{\omega}$, we are faced with some difficulties, which do not appear in classical linking situations (see e.g. [7] for a description of usual linkings). 
The main one comes from assumption (H9), which in some sense is too weak: it would be much easier to find the upper estimate on the min-max levels if we had

$$
F(\varphi) \geqq a_{3}|\varphi|^{2 v},
$$

instead of $F(\varphi) \geqq a_{3}|\varphi \bar{\varphi}|^{v}$ for $\varphi \bar{\varphi}$ large enough.

To deal with this expression $\varphi \bar{\varphi}$, which may vanish even for large values of $|\varphi|$, some technical work has to be done. We start this section by a study of the functional $I^{0}$.

$I^{0}$ splits in two parts, a quadratic one, $\frac{1}{2}\langle\varphi, D \varphi\rangle_{E \times E^{\prime}}$, with

$$
D=-i \gamma^{0} \gamma^{k} \partial_{k}+m \gamma^{0}
$$

and a nonlinear one, $-\int F(\varphi) d^{3} x$. We have the following estimates, for any $\varphi \in \mathbb{C}^{4}$ :

$$
a_{3}|\varphi \bar{\varphi}|^{\nu}-a_{4} \underset{(\mathrm{H} 9)}{\leqq} F(\varphi) \underset{(\mathrm{H} 5)}{\leqq} a_{1}\left(|\varphi|^{\alpha_{1}}+|\varphi|^{\alpha_{2}}\right)
$$

with $a_{1}, a_{3}, a_{4}>0$, and $2<2 v \leqq \max \left(\alpha_{1}, \alpha_{2}\right)<3$. Moreover, we always have $F(\varphi) \geqq 0$.

Let us study $D$. It is a differential operator with constant coefficients. So, in the Fourier domain $\xi=\left(\xi_{1}, \xi_{2}, \xi_{3}\right)$, it becomes the operator of multiplication by the matrix

$$
\hat{D}(\xi)=\xi_{k} \gamma^{0} \gamma^{k}+m \gamma^{0}=\left(\begin{array}{cc}
0 & \xi_{k} \sigma^{k} \\
\xi_{k} \sigma^{k} & 0
\end{array}\right)+\left(\begin{array}{cc}
m & 0 \\
0 & -m
\end{array}\right)
$$

where $\sigma_{1}, \sigma_{2}, \sigma_{3}$ are the Pauli matrices defined in (1.2).

By classical calculations (see e.g. [4]), $\hat{D}(\xi)$ has two eigenvalues, $+\sqrt{m^{2}+\xi^{2}}$ and $-\sqrt{m^{2}+\xi^{2}}$. As a consequence, the spectrum of $D$ is $s p(D)=\mathbb{R} \backslash(-m, m)$.

Note that the meaning of the assumption $|\omega|<m$ already appears: it avoids any degeneracy of $\frac{1}{2}\langle\varphi, D \varphi\rangle_{E \times E^{\prime}}-\frac{\omega}{2}\|\varphi\|_{L^{2}}^{2}$.

Now, there is a natural splitting of $E=H^{1 / 2}\left(\mathbb{R}^{3}, \mathbb{C}^{4}\right)$ as a sum of two Hilbert spaces $E_{+}, E_{-}$, each of them being stable for $D$, with $\operatorname{sp}\left(\left.D\right|_{E_{+}}\right)=[m,+\infty)$ and $s p\left(\left.D\right|_{E_{-}}\right)=(-\infty,-m]$.

Denote $P_{ \pm}$the projection on $E_{ \pm}$with kernel $E_{\mp}$. In the Fourier domain, $P_{ \pm}$ is a multiplication operator, by a matrix $\hat{\pi}_{ \pm}(\xi)$ which is a smooth function of $\xi$. Moreover, $\hat{\pi}_{+}(0)$ is the orthogonal projection from $\mathbb{C}^{4}$ onto $\mathbb{C}^{2} \times\{(0,0)\}$ and $\hat{\pi}_{-}(0)$ is the projection on $\{(0,0)\} \times \mathbb{C}^{2}$.

Consider the following norm on $E$,

$$
\begin{aligned}
\|\varphi\|_{E} & =\left\langle\varphi,\left(D P_{+}-D P_{-}\right) \varphi\right\rangle_{E \times E^{\prime}}^{\frac{1}{2}}=\langle\varphi,|D| \varphi\rangle^{\frac{1}{2}} \\
& =\left(\int_{\mathbb{R}^{3}}\left(\hat{\varphi}(\xi),\left[\hat{D}(\xi) \hat{\pi}_{+}(\xi)-\hat{D}(\xi) \pi_{-}(\xi)\right] \hat{\varphi}(\xi)\right) d^{3} \xi\right)^{\frac{1}{2}} .
\end{aligned}
$$

This norm is equivalent to the classical $H^{1 / 2}$-norm, and for $\|\cdot\|_{E}, E_{+}$and $E_{-}$are orthogonal, as well as for $\|\cdot\|_{L^{2}}$. Moreover, $\forall \varphi \in E, m\|\varphi\|_{L^{2}}^{2} \leqq\|\varphi\|_{E}^{2}$.

Let us call $E^{s}$ the set of functions $\varphi \in E$ of the particular form given by (1.8). $E^{s}$ is a Hilbert subspace of $E$ stable for $D$. So it is stable for $P_{+}$and $P_{-}$, and, denoting $E_{ \pm}^{s}=E_{ \pm} \cap E^{s}$, we have $E^{s}=E_{+}^{s} \oplus E_{-}^{s}$. 
Coming back to $I^{0}$, we now give the key lemma which solves our problem with (H9).

Lemma 2.1. Assume that (H9) is true, and take $\mu>0$ and $N \in \mathbb{N}^{*}$.

There is a radius $R>0$ and an $N$-dimensional vector space $E_{N} \subset E_{+}$such that,

if $\varphi \in E$ satisfies $\left\|P_{-} \varphi\right\|_{E} \leqq R$ and $\left(P_{+} \varphi \in E_{N},\left\|P_{+} \varphi\right\|_{E}=R\right)$,

then $I^{0}(\varphi) \leqq \frac{\mu}{2}\|\varphi\|_{L^{2}}^{2}$.

Moreover, we can impose $E_{N} \subset E_{+}^{s}$.

Note that, in Lemma 2.1, $E_{N}$ and $R$ depend on $\mu, N$. The dependence on $N$ is not very surprising. It would also be true if we had $F(\varphi) \geqq c|\varphi|^{2 v}$. But in that case, we could take $E_{N}, R$ independent of $\mu$ for $N$ fixed. We do not know if the same thing can be done with the estimate $F(\varphi) \geqq c|\varphi \bar{\varphi}|^{v}$, for $|\varphi \bar{\varphi}|$ large enough.

Corollary 2.2. Assume that (H9) is true with $\mu, N, E_{N}, R$ as in Lemma 2.1.

Define $\mathscr{M}_{-}=\left\{\varphi \in E /\left\|P_{-} \varphi\right\|_{E} \leqq R, P_{+} \varphi \in E_{N},\left\|P_{+} \varphi\right\|_{E} \leqq R\right\}$, and $\partial \mathscr{M}_{-}=$ $\left\{\varphi \in \mathscr{M}_{-}\right.$/ either $\left\|P_{-} \varphi\right\|_{E}=R$ or $\left.\left\|P_{+} \varphi\right\|_{E}=R\right\}$.

Then, for any $\varphi \in \partial \mathscr{M}_{-}$and $\omega \in[\mu, m]$, we have $I^{\omega}(\varphi) \leqq 0$.

Proof of the Corollary.

- If $\left\|P_{-} \varphi\right\|_{E}=R$ and $\left\|P_{+} \varphi\right\| \leqq R$, then $I^{\omega}(\varphi) \leqq \frac{1}{2}\left(R^{2}-R^{2}\right)=0$.

- If $\|P-\varphi\|_{E} \leqq R,\left\|P_{+} \varphi\right\|_{E}=R$, we apply Lemma 2.1, and obtain $I^{\omega}(\varphi)=$ $I^{0}(\varphi)-\frac{\omega}{2}\|\varphi\|_{L^{2}}^{2} \leqq \frac{1}{2}(\mu-\omega)\|\varphi\|_{L^{2}}^{2} \leqq 0$.

Proof of Lemma 2.1. We consider the vector $X=\left(\begin{array}{l}1 \\ 0 \\ 0 \\ 0\end{array}\right) \in \mathbb{C}^{4}$. Note that $\gamma^{0} X=$ $X, \hat{D}(0) X=m X, \hat{\pi}_{+}(0) X=X, \hat{\pi}_{-}(0) \gamma^{0} X=\hat{\pi}_{-}(0) X=0$,

$$
\pi_{ \pm}(\xi) \underset{\xi \rightarrow 0}{\rightarrow \rightarrow} \hat{\pi}_{ \pm}(0), \quad \hat{D}(\xi) \underset{\xi \rightarrow 0}{\rightarrow} \hat{D}(0) .
$$

Given $\lambda>0$, we call $V_{\lambda}$ the Hilbert subspace of $E$ of functions $\varphi(x)$ whose Fourier transform may be written $\hat{\varphi}(\xi)=\theta(|\xi|) X$, with $\theta$ arbitrary in $L^{2}\left(\left([0, \lambda], r^{2} d r\right), \mathbb{R}\right)$, and $\theta(r)=0$ if $r \geqq \lambda$.

Clearly, $V_{\lambda}$ is infinite-dimensional and $V_{\lambda} \subset E^{s}$. So $E_{\lambda}=P_{+}\left(V_{\lambda}\right) \subset E_{+}^{s}$, and since $\hat{\pi}_{+}(\xi) X \rightarrow X$ as $\xi \rightarrow 0, E_{\lambda}$ is infinite-dimensional.

Moreover, given $0<\eta<1 / 2$, there is $\lambda(\eta)>0$ such that, if $0<\lambda \leqq \lambda(\eta)$, then for any $\varphi_{\lambda} \in E_{\lambda}$ with $\left\|\varphi_{\lambda}\right\|_{E}=1$ :

$$
\begin{aligned}
& m\left\|\varphi_{\lambda}\right\|_{L^{2}\left(\mathbb{R}^{3}, \mathbb{C}^{4}\right)}^{2} \geqq m\left(\varphi_{\lambda}, \gamma^{0} \varphi_{\lambda}\right)_{L^{2}} \geqq 1-\frac{\eta}{2}, \\
& \left(\forall \varphi_{-} \in E_{-}\right) \quad\left|\left(\varphi_{-}, \gamma^{0} \varphi_{\lambda}\right)_{L^{2}}\right| \leqq \frac{\eta}{2}\left\|\varphi_{-}\right\|_{L^{2}} .
\end{aligned}
$$

Given $N \in \mathbb{N}^{*}$ and $\eta>0$, we select an $N$-dimensional subspace $E_{N}(\eta)$ of $E_{\lambda}(\eta)$. There is $A=A(\eta, N)>0$ such that, for any $e \in E_{N}(\eta)$, with $\|e\|_{E}=1$ :

$$
\begin{gathered}
m\|e\|_{L^{2}\left(\mathbb{R}^{3}, \mathbb{C}^{4}\right)}^{2} \geqq m \int_{[-A,+A]^{3}}\left(e(x), \gamma^{0} e(x)\right) d^{3} x \geqq 1-\eta, \\
\left(\forall \varphi_{-} \in E_{-}\right)\left|\int_{[-A, A]^{3}}\left(e(x), \gamma^{0} \varphi_{-}(x)\right) d^{3} x\right| \leqq \eta\left\|\varphi_{-}\right\|_{L^{2}\left(\mathbb{R}^{3}, \mathbb{C}^{4}\right)} .
\end{gathered}
$$


In the following discussion, we are going to choose $\eta(\mu)>0$ and $R(N, \mu)>0$ such that $E_{N}=E_{N}(\eta(\mu))$ and $R=R(N, \mu)$ satisfy the conclusion of Lemma 2.1.

We consider $\varphi=\varphi_{-}+e, \varphi_{-} \in E_{-}, e \in E_{N},\left\|\varphi_{-}\right\|_{E} \leqq R,\|e\|_{E}=R$. There are two possibilities :

(a) $R^{2}\left(1-\frac{\mu}{2 m}\right) \leqq\left\|\varphi_{-}\right\|_{E}^{2} \leqq R^{2}$. Then

$$
\begin{aligned}
I^{0}(\varphi) & \leqq \frac{1}{2}\langle\varphi, D \varphi\rangle_{E \times E^{\prime}} \\
& =\frac{1}{2}\left(R^{2}-\|\varphi-\|_{E}^{2}\right) \\
& \leqq \frac{\mu R^{2}}{4 m} \leqq \frac{\mu\|e\|_{L^{2}\left(\mathbb{R}^{3}\right)}^{2}}{4(1-\eta)} \\
& \leqq \frac{\mu}{2}\|\varphi\|_{L^{2}\left(\mathbb{R}^{3}\right)}^{2} \text { for } \eta \leqq \frac{1}{2}
\end{aligned}
$$

(b) $\left\|\varphi_{-}\right\|_{E}^{2} \leqq R^{2}\left(1-\frac{\mu}{2 m}\right)$, hence $\left\|\varphi_{-}\right\|_{L^{2}\left(\mathbb{R}^{3}\right)}^{2} \leqq \frac{R^{2}}{m}\left(1-\frac{\mu}{2 m}\right)$. Then

$$
\begin{aligned}
\int_{[-A, A]^{3}}|\varphi \bar{\varphi}|^{v} d^{3} x \geqq & \frac{1}{(2 A)^{3(v-1)}} \cdot\left|\int_{[-A, A]^{3}}(\varphi \bar{\varphi}) d^{3} x\right|^{v} \\
\geqq & \frac{1}{(2 A)^{3(v-1)}} \mid \int_{[-A, A]^{3}} d^{3} x\left(e, \gamma^{0} e\right)+2 \iint_{[-A, A]^{3}} d^{3} x\left(e, \gamma^{0} \varphi_{-}\right) \\
& +\left.\int_{[-A, A]^{3}} d^{3} x\left(\varphi_{-}, \gamma^{0} \varphi-\right)\right|^{v} \\
\geqq & \frac{1}{(2 A)^{3(v-1)}}\left[R^{2} \frac{1-\eta}{m}-2 R \eta\left\|\varphi_{-}\right\|_{L^{2}\left(\mathbb{R}^{3}\right)}-\left\|\varphi_{-}\right\|_{L^{2}\left(\mathbb{R}^{3}\right)}^{2}\right]^{v} \\
\geqq & \frac{1}{(2 A)^{3(v-1)}}\left[(1-\eta)-2 \eta \sqrt{m-\mu / 2}-\left(1-\frac{\mu}{2 m}\right)\right]^{v} \cdot \frac{R^{2 v}}{m^{v}} \\
\geqq & \frac{1}{(2 A)^{3(v-1)}}\left[\frac{\mu}{2 m}-\eta(1+2 \sqrt{m})\right]^{v} \frac{R^{2 v}}{m^{v}} \\
\geqq & \frac{1}{(2 A)^{3(v-1)}} \cdot\left(\frac{\mu}{4 m^{2}}\right)^{v} \cdot R^{2 v}, \quad \text { if } \eta \leqq \frac{\mu}{4 m(1+2 \sqrt{m})} \cdot
\end{aligned}
$$

So

$$
\begin{aligned}
I^{0}(\varphi) & \leqq \frac{1}{2}\|e\|_{E}^{2}-\left(\int_{[-A, A]^{3}} a_{3}|\varphi \bar{\varphi}|^{v}\right)+(2 A)^{3} a_{4} \\
& \leqq \frac{R^{2}}{2}-K_{1} R^{2 v}+K_{2}<0 \quad \text { if } \quad R \geqq \bar{R}\left(\mu, A, a_{3}, a_{4}\right) .
\end{aligned}
$$

Finally, we take $\eta(\mu)=\min \left(\frac{1}{2}, \frac{\mu}{4 m(1+2 \sqrt{m})}\right), R(N, \mu)=\bar{R}$, and Lemma 2.1 is proved. 
We now describe the linkings of Theorems 1 and 3 .

A. The linking of Theorem 1. In this part, we assume that $F$ satisfies (H5), (H6) and (H9). We suppose, moreover, the following :

$F$ can be written in the form $F(\varphi)=H\left(\left|\varphi_{1}\right|^{2},\left|\varphi_{2}\right|^{2}\right)$, with $\varphi_{i} \in \mathbb{C}^{2}, \varphi=\left(\begin{array}{c}\varphi_{1} \\ \varphi_{2}\end{array}\right) \in \mathbb{C}^{4}$, arbitrary, $H$ of class $C^{2}$.

Note that these conditions are fulfilled in particular when $F$ is of the form (1.7), with $G$ satisfying $(\mathrm{H} 1,2,3)$.

Let us now define $\nabla I^{\omega}$ as the gradient of $I^{\omega}$ for $\|\cdot\|_{E}$-scalar product, that is, $\nabla I^{\omega}=|D|^{-1}\left(I^{\omega}\right)^{\prime}$. As a consequence of (H10), if $\varphi \in E^{s}$, then $\nabla I^{\omega}(\varphi) \in E^{s}$, and a critical point of $\left.I^{\omega}\right|_{E^{s}}$ is also critical point of $I^{\omega}$. Moreover, $I^{\omega}$ is even.

Given $0<\mu<\frac{m}{2}$ and $N \in \mathbb{N}^{*}$, we consider $\mathscr{M}_{-}$defined in Corollary 2.1, and we define $\mathscr{M}_{-}^{s}=\mathscr{M}_{-} \cap E^{s}, \partial \mathscr{M}_{-}^{s}=\partial \mathscr{M}_{-} \cap E^{s} \subset \mathscr{M}_{-}^{s}$ (we have imposed $E_{N} \subset E_{+}^{s}$ in Lemma 2.1).

From (H5), there are $0<r(\mu)<R(N, \mu)$ and $\rho(\mu)>0$ such that

$$
\left(\omega \leqq m-\mu \Longrightarrow \inf I^{\omega}\left(\Sigma_{+}^{s}\right) \geqq \rho\right) \text {, }
$$

with

$$
\Sigma_{+}^{s}=\left\{\varphi \in E_{+}^{s} /\|\varphi\|_{E}=r(\mu)\right\} .
$$

We define the flow $h_{t}^{\omega}, t \geqq 0$ of $\left.\nabla I^{\omega}\right|_{E^{s}}$ by

$$
\left\{\begin{array}{l}
h_{0}^{\omega}=I d_{E^{s}} \\
\frac{\partial}{\partial t} h_{t}^{\omega}=\nabla I^{\omega} \circ h_{t}^{\omega} .
\end{array}\right.
$$

$\nabla I^{\omega}$ being locally Lipschitz, $h_{t}^{\omega}$ is well-defined and continuous.

Moreover, $I^{\omega} \circ h_{t}^{\omega}(\varphi)$ increases as $t$ increases, $\omega, \varphi$ fixed, so Corollary $2.2 \mathrm{im}$ plies that $h_{t}^{\omega}\left(\Sigma_{+}^{s}\right) \cap \partial \mathscr{M}_{-}^{s}=\emptyset \quad \forall t \geqq 0 \quad \forall \omega \in[\mu, m-\mu]$. Finally, we remark that $\nabla I^{\omega}(\varphi)=P_{+} \varphi-P_{-} \varphi+k^{\omega}(\varphi)$, where $k^{\omega}$ is odd, non-linear, continuous, and maps bounded subsets of $E^{s}$ onto precompact subsets of $E^{s}$.

So, by classical arguments (see e.g. $[1,3]$ ), we have the following result:

Lemma 2.3. We assume that $(\mathrm{H} 5,6,9,10)$ are true.

To $0<\mu<\frac{m}{2}$ and $N \in \mathbb{N}^{*}$, we associate $R, \mathscr{M}_{-}^{s}, \partial \mathscr{M}_{-}^{s}, r, \rho, \Sigma_{+}^{s}$ as above.

Then, for any $\omega \in[\mu, m-\mu]$ and $t \geqq 0$,

$$
\gamma\left[h_{t}^{\omega}\left(\Sigma_{+}^{s}\right) \cap \mathscr{M}_{-}^{s}\right] \geqq N,
$$

where $\gamma$ is the genus (or $\mathbf{Z}_{2}$-degree) of symmetric sets.

Lemma 2.3 has an immediate consequence:

Corollary 2.4. With the assumptions and notations of Lemma 2.3, for $\omega$ fixed in $[\mu, m-\mu]$, we can define

$$
\begin{aligned}
& c_{N}(\omega)=\inf \left\{\sup I^{\omega}(X) / X=-X \subset E^{s},\right. \\
& \left.\quad \text { and }(\forall t \geqq 0) \quad \gamma\left(h_{t}^{\omega}\left(\Sigma_{+}^{s}\right) \cap X\right) \geqq N\right\} .
\end{aligned}
$$


If $N_{1}<N_{2}$, then $c_{N_{1}}(\omega) \leqq c_{N_{2}}(\omega)$, and we have the estimates

$$
0<\rho \leqq c_{N}(\omega) \leqq \Gamma_{N}<+\infty,
$$

where $\rho$ depends only on $\left(a_{1}, \alpha_{1}, \alpha_{2}, \mu\right)$ and $\Gamma_{N}$ on $\left(a_{3}, a_{4}, v, \mu, N\right)$.

Moreover, there is a sequence $\left(\varphi_{n}\right)_{n \geqq 0}, \varphi_{n} \in E^{s}$, such that

$$
I^{\omega}\left(\varphi_{n}\right) \rightarrow c_{N}(\omega)
$$

and

$$
\left(I^{\omega}\right)^{\prime}\left(\varphi_{n}\right) \underset{E^{\prime}}{\rightarrow} 0 \text { as } n \rightarrow+\infty
$$

Proof of the Corollary. The monotonicity of $\left(c_{N}(\omega)\right)_{N \geqq 1}$ is obvious.

In the estimate $\rho \leqq c_{N}(\omega), \rho$ is the same as in Lemma 2.3. It satisfies inf $I^{m-\mu}\left(\Sigma_{+}^{s}\right) \geqq \rho$. For $c_{N}(\omega) \leqq \Gamma_{N}$, we choose $\Gamma_{N}=\sup I^{\mu}\left(\mathscr{M}_{-}\right)$, where $\mathscr{M}_{-}$is associated to $\mu, N$ as in Corollary 2.2. The existence of $\left(\varphi_{n}\right)_{n \geqq 0}$ is classical result of min-max theory (see e.g. [1]).

B. The Linking of Theorem 3. In this section, no symmetry assumption is made on $F$, and we cannot use the space $E^{s}$. In $E$, the gradient of $I^{\omega}$ is of the form $P_{+} \varphi-$ $P_{-} \varphi+k^{\omega}(\varphi)$, as in part A, but $k$ does not send bounded sets into precompact sets. The reason for that is the invariance of the problem by translations in $\mathbb{R}^{3}$. Such a situation has been studied, in the context of homoclinic orbits of Hamiltonian systems, by Hofer and Wysocki (see [11]), and we will use their ideas to overcome this difficulty. See also Tanaka [19] for another approach.

We suppose that $F$ satisfies conditions (H5,6,9). Let $0<\mu<\frac{m}{2}$ be fixed. We consider $R, E_{1}$ associated to $\mu$ and $N=1$ as in Lemma 2.1. We take $e_{+} \in E_{1}$, with $\left\|e_{+}\right\|_{E}=1$.

We define

$$
\mathscr{N}_{-}=\left\{\varphi=\varphi_{-}+\lambda e_{+} / \varphi_{-} \in E_{-},\left\|\varphi_{-}\right\|_{E} \leqq R, \lambda \in[0, R]\right\}
$$

and

$$
\partial \mathscr{N}_{-}=\left\{\varphi=\varphi_{-}+\lambda e_{+} \in \mathscr{N}_{-} / \text {either }\left\|\varphi_{-}\right\|_{E}=R \text { or } \lambda \in\{0, R\}\right\} .
$$

Since $I^{\omega} \leqq 0$ on $E_{-}$, it follows from Corollary 2.2 that $I^{\omega} \leqq 0$ on $\partial \mathscr{N}_{-}$.

We also define $\Sigma_{+}=\left\{\varphi \in E_{+} /\|\varphi\|_{E}=r\right\}$, where $0<r<R$ is chosen such that, for any $\varphi \in E_{+}$with $\|\varphi\|_{E} \leqq r, I^{m-\mu}(\varphi) \geqq 0$, and that $\rho=\inf _{\Sigma_{+}} I^{m-\mu}>0$. This is possible thanks to (H5).

We call $\eta_{t}^{\omega}$ the flow of $-\nabla I^{\omega}=-|D|^{-1}\left(I^{\omega}\right)^{\prime}$ at time $t \geqq 0$, defined by

$$
\left\{\begin{array}{l}
\eta_{0}^{\omega}=\quad I d_{E} \\
\partial_{t} \eta_{t}^{\omega}=-\nabla I^{\omega} \circ \eta_{t}^{\omega}, \quad \omega \in[\mu, m-\mu]
\end{array} .\right.
$$

We start with the following lemma:

Lemma 2.5. (H5, 6, 9) are supposed to be true, $\mu \in\left(0, \frac{m}{2}\right)$ is fixed, $\Sigma_{+}, \mathscr{N}_{-}, h_{t}^{\omega}$ are defined as above and $\omega \in[\mu, m-\mu]$. Consider the mapping

$$
\begin{aligned}
H^{\omega}: \mathbb{R}_{+} \times \mathcal{N}_{-} & \rightarrow E_{-} \times \mathbb{R}_{+} \\
(t, \varphi) & \mapsto\left(P_{-} \circ \eta_{t}^{\omega}(\varphi),\left\|P_{+} \circ \eta_{t}^{\omega}(\varphi)\right\|_{E}^{2}\right)
\end{aligned}
$$


Then $H^{\omega}$ is a Fredholm mapping of class $C^{1}$ and index 1, and there is $\varepsilon>0$ such that for any $T \geqq 0$, the restriction of $H^{\omega}$ to $U_{\varepsilon, T}=$ $\left(H^{\omega}\right)^{-1}\left(B_{-}(\varepsilon) \times\left[(r-\varepsilon)^{2},(r+\varepsilon)^{2}\right]\right) \cup\left([0, T] \times \mathscr{N}_{-}\right)$is proper.

Proof. We just give a sketch of proof. For similar (and more detailed) arguments, we refer to [11], Lemma 4.4, and [15], Lemma 4.4.

We may write

$$
\nabla I^{\omega}(\varphi)=P_{+} \varphi-P_{-} \varphi-\omega|D|^{-1} \varphi-|D|^{-1} F^{\prime}(\varphi),
$$

and $\varphi \mapsto|D|^{-1} F^{\prime}(\varphi)$ has a compact differential

$$
\chi \in E \mapsto|D|^{-1}\left(F^{\prime \prime}(\varphi) \cdot \chi\right) \in E \quad \text { at any point } \varphi \in E .
$$

So, by variation of the constant,

$$
\eta_{t}^{\omega}(\varphi)=\exp \left\{t\left(P_{-}-P_{+}+\omega|D|^{-1}\right)\right\} \cdot \varphi+K_{t}(\varphi)=L_{t} \varphi+K_{t}(\varphi),
$$

$K_{t}: E \rightarrow E$ is of class $C^{1}$ and has a compact differential at any $\varphi \in E$. Moreover, $K_{t}$ is compact from $E$ to $H_{l o c}^{\frac{1}{2}}$.

As a consequence, $\eta_{t}^{\omega}, H^{\omega}$ are Fredholm mappings of class $C^{1}, \eta_{t}^{\omega}$ has index 0 , and $H^{\omega}$ has index 1 .

Given $\varepsilon>0$, let us take a sequence $\left(t_{n}, \varphi_{n}\right)$ in $U_{\varepsilon, T}$ such that $H^{\omega}\left(t_{n}, \varphi_{n}\right)=$ $\left(\psi_{n}, r_{n}^{2}\right)$ has a limit $\left(\psi_{*}, r_{*}^{2}\right)$ as $n$ goes to infinity. To end the proof of Lemma 2.5 , we have to show that for $\varepsilon$ small enough, $\left(\varphi_{n}\right)$ is precompact in $E$.

After extraction, we may assume that $t_{n}$ tends to $t_{*} \in[0, T]$. We have $\varphi_{n}=$ $L_{-t_{n}}\left(\psi_{n}+r_{n} e_{+}\right)-L_{-t_{n}} K_{t_{n}}\left(\varphi_{n}\right)=\varphi_{n}^{1}+\varphi_{n}^{2}$. The sequence $\left(\varphi_{n}^{1}\right)$ has a limit $\varphi_{*}^{1}$ for the topology of $E$, and after a new extraction, there is $\varphi_{*}^{2} \in E$ such that $\beta \varphi_{n}^{2} \rightarrow \beta \varphi_{*}^{2}$ for any compactly supported function $\beta \in C^{\infty}(\mathbb{R}, \mathbb{R})$.

Let us denote $\varphi_{n}^{3}=\varphi_{n}^{2}-\varphi_{*}^{2}$. It is not very difficult to prove that $P_{-} \circ \eta_{t_{n}}^{\omega}\left(\varphi_{n}^{3}\right)$ $\rightarrow 0$ and that $\left\|\eta_{t_{n}}^{\omega}\left(\varphi_{n}^{3}\right)\right\|_{E}$ is smaller than $r+2 \varepsilon$ for $n$ large enough. So, choosing $\varepsilon$ small enough, we find that

$$
\lim \inf I^{\omega}\left(\varphi_{n}^{3}\right) \geqq \lim \inf I^{\omega} \circ \eta_{t_{n}}^{\omega}\left(\varphi_{n}^{3}\right) \geqq 0 .
$$

Since $P_{+}\left(\varphi_{n}^{3}\right) \rightarrow 0$ as $n \rightarrow \infty$, this inequality implies that $\varphi_{n}^{3} \rightarrow 0$ for the topology of $E$. The sequence $\varphi_{n}$ is thus convergent in $\mathrm{E}$, and Lemma 2.5 is proved.

Lemma 2.5 allows to work with a generalized version of the Leray-Schauder $\mathrm{Z}_{2}$ degree, due to Smale [16]. Given $T \geqq 0$, we may define $V_{\varepsilon, T}=U_{\varepsilon, T} \cap\left(\{T\} \times \mathscr{N}_{-}\right)$ and $d_{T}=\operatorname{deg}\left(H^{\omega}(T, \cdot), V_{\varepsilon, T},\left(0, r^{2}\right)\right)$.

We have $d_{0}=1$. From Corollary 2.2 and Lemma 2.5, $d_{T}=d_{0}$. As a consequence, the equation $H^{\omega}(T, \varphi)=\left(0, r^{2}\right)$ has always a solution in $V_{\varepsilon, T}$, and we have the following result

Corollary 2.6. With the assumptions and notations of Lemma 2.5, for any $t \geqq 0$, $\eta_{t}^{\omega}\left(\mathscr{N}_{-}\right) \cap \Sigma_{+}$is non-empty.

So, if we define $c(\omega)=\inf _{t \geqq 0} \sup I^{\omega} \circ \eta_{t}^{\omega}\left(\mathscr{N}_{-}\right)$, we have the estimates

$$
0<\rho \leqq c(\omega) \leqq \Gamma<+\infty,
$$

where $\rho$ depends only on $a_{1}, \alpha_{1}, \alpha_{2}, \mu$, and $\Gamma$ on $a_{3}, a_{4}, v, \mu$. 
Moreover, there is a sequence $\left(\varphi_{n}\right)_{n \geqq 0}, \varphi_{n} \in E$, such that $I^{\omega}\left(\varphi_{n}\right) \rightarrow c(\omega)$ and $\left\|\left(I^{\omega}\right)^{\prime}\left(\varphi_{n}\right)\right\|_{E^{\prime}} \rightarrow 0$, as $n \rightarrow \infty$.

\section{Section 3. Estimates and Auxiliary Results}

We start with a priori estimates for the solutions of the nonlinear Dirac equation (1.5), obtained via the so-called virial theorem. These estimates do not give direct a priori bounds on the norm of the solution in $\mathrm{E}$. The indefiniteness of the quadratic part of the energy functional $I^{\omega}$ (and even of the two pieces of it, the one containing the derivatives and the $0^{\text {th }}$-order term) is one of the main causes for that. But the nonlinear part makes things much worse. Indeed, even when $F$ satisfies (1.7), we see that the term $\bar{\varphi} \varphi$ has a cancellation cone, and hence for any $G, G(\bar{\varphi} \varphi)$ can never have good growth properties: it can be equal to 0 for small $|\varphi|$ and for large $|\varphi|$ as well.

Proposition 3.1. Let $F \in C^{1}\left(\mathbb{C}^{4}, \mathbb{R}\right)$ and let $\varphi \in H^{1}\left(\mathbb{R}^{3}, \mathbb{C}^{4}\right)$ a solution of $(1.5)$ such that $F(\varphi) \in L^{1}\left(\mathbb{R}^{3}\right)$. Then $\varphi$ satisfies

$$
\int_{\mathbb{R}^{3}}\left(i \gamma^{0} \gamma^{k} \partial_{k} \varphi, \varphi\right) d x=\frac{3}{2} \int_{\mathbb{R}^{3}}\left(m(\bar{\varphi} \varphi)-\omega|\varphi|^{2}-2 F(\varphi)\right) d x
$$

Remark. For the nonlinear Dirac equation (1.5), the above identity (3.1) is the equivalent of the so-called Pohozaev identity for semilinear elliptic equations. Formally (3.1) derives from the fact that if $\varphi$ is a critical point of $I^{\omega}$, then

$$
\left.\frac{d}{d t} I^{\omega}\left(\varphi\left(\frac{\dot{-}}{\bar{t}}\right)\right)\right|_{t=1}=0
$$

Proof of Proposition 3.1. We notice that the generator of the group $t \rightarrow \varphi(\dot{\bar{t}})$ is $x \cdot \nabla \varphi$. We use this multiplier to prove (3.1).

Multiplying Eq. (1.5) by $\gamma^{0}$ and taking the scalar product of this with $x \cdot \nabla \varphi$ we obtain for all $R>0$,

$$
\int_{B_{R}}\left(i \gamma^{0} \gamma^{k} \partial_{k} \varphi, x^{j} \partial_{j} \varphi\right) d x=\int_{B_{R}}\left(\nabla A(\varphi), x^{j} \partial_{j} \varphi\right) d x
$$

where by $\nabla A(\varphi)$ we denote $-m \gamma^{0} \varphi+\omega \varphi+F^{\prime}(\varphi)$.

Integrating by parts in $B_{R}=\left\{x \in \mathbb{R}^{3} ;|x|<R\right\}$ we obtain

$$
\int_{B_{R}}\left(\nabla A(\varphi), x^{j} \partial_{j} \varphi\right) d x=-3 \int_{B_{R}} A(\varphi) d x+\int_{S_{R}} A(\varphi)(x \cdot n) d \sigma,
$$

where $S_{R}=\left\{x \in \mathbb{R}^{3} ;|x|=R\right\}$. Moreover

$$
\begin{aligned}
& \operatorname{Re} \int_{B_{R}}\left(i \gamma^{0} \gamma^{k} \partial_{k} \varphi, x^{j} \partial_{j} \varphi\right) d x=-\int_{B_{R}}\left(i \gamma^{0} \gamma^{k} \partial_{k} \varphi, \varphi\right) d x \\
& \quad+\frac{1}{2} \int_{S_{R}}\left[\left(i \gamma^{0} \gamma^{k} \partial_{k} \varphi, \varphi\right)(x \cdot n)+\left(x^{j} \partial_{J} \varphi, i n_{k} \gamma^{0} \gamma^{k} \varphi\right)\right] d \sigma
\end{aligned}
$$

where $n=\left(n_{1}, n_{2}, n_{3}\right)$ is the outward unit vector to $S_{R}$. 
Therefore, since $\bar{\varphi} \varphi,|\varphi|^{2}$ and $F(\varphi) \in \mathbb{R}$,

$$
\begin{aligned}
& \left|\int_{B_{R}}\left(\left(i \gamma^{0} \gamma^{k} \partial_{k} \varphi, \varphi\right)-\frac{3}{2} m(\bar{\varphi} \varphi)+\frac{3}{2} \omega|\varphi|^{2}-3 F(\varphi)\right)\right| \\
& \quad \leqq R \int_{S_{R}}(|A(\varphi)|+|\nabla \varphi||\varphi|) d \sigma .
\end{aligned}
$$

Now, since $\varphi \in H^{1}\left(\mathbb{R}^{3}\right)$ and $F(\varphi) \in L^{1}\left(\mathbb{R}^{3}\right)$, there exists a sequence $R_{n} \rightarrow+\infty$ such that

$$
R_{n} \int_{S_{R_{n}}}\left(|\nabla \varphi||\varphi|+|\varphi|^{2}+|F(\varphi)|\right) d \sigma \underset{n \rightarrow+\infty}{\rightarrow} 0
$$

Then we take $R=R_{n}$ in (3.6) and prove (3.1).

Let us now see why a solution of Eq. (1.5) which is in $H^{1 / 2}\left(\mathbb{R}^{3}, \mathbb{C}^{4}\right)$ has more regularity. This is done by using a standard bootstrap argument and the regularizing properties of the inverse of the linear Dirac operator.

Proposition 3.2. We suppose that $F$ satisfies (H5, 6, 7, 8), and we take $\omega \in(0, m)$. If $\varphi \in E$ is a solution of (1.5) such that

$$
\left|I^{\omega}(\varphi)\right| \leqq \Gamma \quad \text { and } \quad\|\varphi\|_{L^{2}\left(\mathbb{R}^{3}, \mathbb{C}^{4}\right)} \leqq K,
$$

then for any $q \in[2,+\infty)$,

$$
\varphi \in W^{1, q}\left(\mathbb{R}^{3}\right) \text { and }\|\varphi\|_{W^{1, q}} \leqq M_{q},
$$

where $M_{q}$ depends only on the constants $a_{1}, a_{2}, C_{\delta}, \alpha_{1}, \alpha_{2}, \alpha, \beta$, which appear in (H5, 6, 7, 8), and on $m, \Gamma, K$ (and $q$, of course).

Proof. From (1.5), we write

$$
\varphi=D^{-1}\left(F^{\prime}(\varphi)+\omega \varphi\right) \text {, with } D=-i \gamma^{0} \gamma^{k} \partial_{k}+m \gamma^{0} .
$$

From (H5), (H7) and (1.5), we have

$$
0 \leqq(\alpha-2) \int_{\mathbb{R}^{3}} F(\varphi) d^{3} x \leqq 2 I^{\omega}(\varphi) \leqq 2 \Gamma
$$

From (H8), for any $x \in \mathbb{R}^{3}$,

$$
\left|F^{\prime}(\varphi)+\omega \varphi\right|(x) \leqq\left(a_{3}+m\right)\left(1+|F(\varphi)|^{\frac{1}{\beta}}\right)|\varphi|(x) .
$$

By assumption, $\|\varphi\|_{L^{2}} \leqq K$. We want to improve this estimate iteratively. Assume that we have obtained $\|\varphi\|_{L^{q}} \leqq K_{q}$ for some $q \geqq 2$.

Then, by Hölder's inequality,

$$
\left\|\left.F(\varphi)\right|^{\frac{1}{\beta}} \mid \varphi\right\|_{L^{p}} \leqq\|F(\varphi)\|_{L^{1}}^{1 / \beta} \cdot\|\varphi\|_{L^{q}} \leqq\left(\frac{2 \Gamma}{\alpha-2}\right)^{\frac{1}{\beta}} K_{q},
$$

with $\frac{1}{p}=\frac{1}{q}+\frac{1}{\beta}$.

So $\varphi=\varphi_{1}+\varphi_{2}$, with

$$
\left\|\varphi_{1}\right\|_{W^{1,2}}+\left\|\varphi_{1}\right\|_{W^{1, q}} \leqq M_{q, 1} \text { and }\left\|\varphi_{2}\right\|_{W^{1, p}} \leqq M_{p, 2} \text {. }
$$


So $\|\varphi\|_{W^{1, p}} \leqq M_{p}$. Hence

$$
\|\varphi\|_{L^{q^{\prime}}} \leqq K_{q^{\prime}} \quad \text { with } \frac{1}{q^{\prime}}=\frac{1}{p}-\frac{1}{3}=\frac{1}{q}-\left(\frac{1}{3}-\frac{1}{\beta}\right)
$$

if this quantity is positive. Continuing this process, we prove the proposition.

Proposition 3.3. Assume that $F: \mathbb{C}^{4} \rightarrow \mathbb{R}$ satisfies $(\mathrm{H} 5,6,7,8)$. Then for every pair of constants $c_{1}, c_{2}$ there exists a constant $M$, depending only on $c_{1}, c_{2}$, such that any solution $\varphi \in E$ of (1.5) for which

$$
c_{1} \leqq I^{\omega}(\varphi) \leqq c_{2},
$$

satisfies

$$
\begin{gathered}
\left|\int_{\mathbb{R}^{3}}\left(i \gamma^{0} \gamma^{k} \partial_{k} \varphi, \varphi\right) d x\right| \leqq M, \\
\left|\int_{\mathbb{R}^{3}}\left(m(\bar{\varphi} \varphi)-\omega|\varphi|^{2}\right) d x\right| \leqq M, \\
\left|\int_{\mathbb{R}^{3}} F(\varphi) d x\right| \leqq M .
\end{gathered}
$$

Remark. The quadratic forms defined by the integrals in (3.8) and in (3.9) are not definite positive or negative. Moreover, the subspaces of $H^{1 / 2}$ on which these forms are either positive or negative definite are all infinite dimensional. Therefore (3.8) and (3.9) do not provide a priori estimates for the solutions of Eq. (1.5) in any Sobolev space. Inequality (3.10) could provide one if for instance $F$ behaved like the sum of power functions at infinity. Nevertheless in the often encountered case when $F$ depends on $\varphi$ through the function $\bar{\varphi} \varphi$ or a similar one, (3.10) again does not provide any estimate.

Proof of Proposition 3.3. First of all, Proposition 3.2 implies that $\varphi \in H^{1}$, and from (H5), $F(\varphi) \in L^{1}$, so we can apply Proposition 3.1 to $\varphi$.

We multiply Eq. (1.5) by $-\varphi^{*}$ on the right and $\gamma^{0}$ on the left. We obtain

$$
\int_{\mathbb{R}^{3}}\left[\left(-i \gamma^{0} \gamma^{k} \partial_{k} \varphi, \varphi\right)+m(\bar{\varphi} \varphi)-\omega|\varphi|^{2}-(\nabla F(\varphi), \varphi)\right] d x=0 .
$$

From (3.1) and (3.7) we get

$$
-6 c_{2} \leqq \int_{\mathbb{R}^{3}}\left(i \gamma^{0} \gamma^{k} \partial_{k} \varphi, \varphi\right) d x \leqq-6 c_{1},
$$

which of course implies (3.8). Now, from (H5) and (H7) we have

$$
\int_{\mathbb{R}^{3}}(\nabla F(\varphi), \varphi) \geqq 0 .
$$

Therefore, (3.11)-(3.13) imply

$$
\int_{\mathbb{R}^{3}}\left(m(\bar{\varphi} \varphi)-\omega|\varphi|^{2}\right) d x \geqq-6 c_{2} .
$$


Next we multiply (3.11) by -2 and (3.7) by $2 \alpha$ and add them. Then we have

$$
\begin{aligned}
& (\alpha-2) \int_{\mathbb{R}^{3}}\left(m(\bar{\varphi} \varphi)-\omega|\varphi|^{2}\right) d x \\
& \leqq(\alpha-2) \int_{\mathbb{R}^{3}}\left(i \gamma^{0} \gamma^{k} \partial_{k} \varphi, \varphi\right) d x+2 \alpha \int_{\mathbb{R}^{3}} F(\varphi) d x \\
& \quad+2 \alpha c_{2}-2 \int(\nabla F(\varphi), \varphi) d x \\
& \leqq(\alpha-2) \int_{\mathbb{R}^{3}}\left(i \gamma^{0} \gamma^{k} \partial_{k} \varphi, \varphi\right) d x+2 \alpha c_{2} \quad \text { by (H7)., }
\end{aligned}
$$

This and (3.7), (3.12) prove (3.9) and (3.10).

Let us define two kinds of norms, that will be used in the sequel.

\section{Definition 3.4.}

- Given $q \geqq 1$, and $\varphi: \mathbb{R}^{3} \rightarrow B$ measurable, where $(B,|\cdot|)$ is a Banach space, we write

$$
U_{q}(\varphi)=\sup _{l \in \mathbf{Z}^{3}}\left(\int_{Q_{l}}|\varphi(x)|^{q} d x\right)^{\frac{1}{q}},
$$

where $Q_{l}=\left\{\left(x^{1}, x^{2}, x^{3}\right) \in \mathbb{R}^{3} / l^{i} \leqq x^{i} \leqq l^{l}+1, \quad \forall i\right\}$.

$U_{q}$ is a norm on the Banach space "uniform $L_{\text {loc }}^{q}\left(\mathbb{R}^{3}, B\right)$."

- Given $q \geqq 1$, and $\varphi: \mathbb{R}^{3} \rightarrow B$ measurable, we define

$$
N_{q}(\varphi)=\left(\sum_{l \in \mathbf{Z}^{3}}\left[\int|\varphi|^{q} d x\right]^{\frac{2}{q}}\right)^{\frac{1}{2}} .
$$

$N_{q}$ is a norm on the Banach space of functions for which it is finite. Note that $N_{2}=\|\cdot\|_{L^{2}\left(\mathbb{R}^{3}, B\right) \text {. }}$

The following result will be very useful in Sect. 5:

Proposition 3.5. Let $\omega \in(0, m)$ and $\beta \in(3, \infty)$. There are $K>0$ and $n_{0} \geqq 1$, which depend only on $\omega, m, \beta$, such that if $\varphi \in E, \quad \chi \in$ "uniform $L_{\text {loc }}^{\beta}\left(\mathbb{R}^{3}, \mathbb{R}\right)$," $\Omega \in E^{\prime}$ and $\psi: \mathbb{R}^{3} \rightarrow \mathbb{C}^{4}$ measurable, satisfy $D \varphi-\omega \varphi=\psi+\Omega$, with $|\psi(x)| \leqq$ $\chi(x) \cdot|\varphi(x)|$ for a.e. $x \in \mathbb{R}^{3}$, then

$$
\|\varphi\|_{E} \leqq K\left(U_{\beta}(\chi)^{n_{0}}\|\varphi\|_{L^{2}\left(\mathbb{R}^{3}\right)}+\left[1+U_{\beta}(\chi)\right]^{n_{0}-1}\|\Omega\|_{E^{\prime}}\right) .
$$

Proof. We denote $\varphi_{1}=(D-\omega I)^{-1} \psi, \varphi_{2}=(D-\omega I)^{-1} \Omega$. We know that $N_{2}(\varphi)=$ $\|\varphi\|_{L^{2}}$ is finite. We follow the same iterative scheme as in the proof of Proposition 3.2 .

If, for some $q \geqq 2, N_{q}(\varphi)$ is finite, then

$$
N_{p}(\chi \varphi) \leqq U_{\beta}(\chi) N_{q}(\varphi) \text { with } \frac{1}{p}=\frac{1}{q}+\frac{1}{\beta},
$$

by Hölder's inequality. So,

$$
N_{p}\left(\varphi_{1}\right)+N_{p}\left(\nabla \varphi_{1}\right) \leqq C_{p}^{(0)} U_{\beta}(\chi) N_{q}(\varphi)
$$


and by Sobolev embedding,

$$
N_{q^{\prime}}\left(\varphi_{1}\right) \leqq C_{q}^{(1)} U_{\beta}(\chi) N_{q}(\varphi),
$$

$\frac{1}{q^{\prime}}=\frac{1}{p}+\frac{1}{3}=\frac{1}{q}+\left(\frac{1}{\beta}-\frac{1}{3}\right)$, provided this quantity is positive. We also have

$$
N_{3}\left(\varphi_{2}\right) \leqq C^{(2)}\left\|\varphi_{2}\right\|_{E} \leqq C^{(3)}\|\Omega\|_{E^{\prime}},
$$

and $N_{q^{\prime}}\left(\varphi_{2}\right) \leqq N_{3}\left(\varphi_{2}\right)$ if $q^{\prime} \leqq 3$, hence

$$
N_{q^{\prime}}(\varphi) \leqq C_{q}^{(4)}\left(U_{\beta}(\chi) N_{q}(\varphi)+\|\Omega\|_{E^{\prime}}\right), \quad \text { if } q^{\prime} \leqq 3 .
$$

Starting with $q_{0}=2$, we iterate the process, until $q_{n_{0}} \geqq 3$, i.e. $p_{n_{0}} \geqq \frac{3}{2}$. By induction on $n$, we get

$$
N_{q_{n_{0}-1}}(\varphi) \leqq C^{(5)}\left[U_{\beta}(\chi)^{n_{0}-1} \cdot\|\varphi\|_{L^{2}}+\left(1+U_{\beta}(\chi)\right)^{n_{0}-2} \cdot\|\Omega\|_{E^{\prime}}\right] .
$$

So

$$
\begin{aligned}
N_{\frac{3}{2}}\left(\varphi_{1}\right) & +N_{\frac{3}{2}}\left(\nabla \varphi_{1}\right) \leqq N_{p_{n_{0}}}\left(\varphi_{1}\right)+N_{p_{n_{0}}}\left(\nabla \varphi_{1}\right) \\
& \leqq C^{(6)} U_{\beta}(\chi)\left(U_{\beta}(\chi)^{n_{0}-1}\|\varphi\|_{L^{2}}+\left(1+U_{\beta}(\chi)\right)^{n_{0}-2} \cdot\|\Omega\|_{E^{\prime}}\right) \\
& \leqq C^{(7)}\left[U_{\beta}(\chi)^{n_{0}}\|\varphi\|_{L^{2}}+\left(1+U_{\beta}(\chi)\right)^{n_{0}-1} \cdot\|\Omega\|_{E^{\prime}}\right] .
\end{aligned}
$$

We have the Sobolev inequality

$$
\left\|\varphi_{1}\right\|_{E} \leqq C^{(8)}\left(\left|\varphi_{1}\right|_{\frac{3}{2}}+\left|\nabla \varphi_{1}\right|_{\frac{3}{2}}\right) .
$$

Moreover,

$$
\left\|\varphi_{2}\right\|_{E} \leqq C^{(9)}\|\Omega\|_{E^{\prime}}
$$

hence the proposition.

Proposition 3.5 has the following consequence:

Corollary 3.6. Assume that $F$ satisfies $(\mathrm{H} 5,6,8)$, and take $\omega \in(0, m)$.

Then there are $\eta_{0}>0$ and $K_{0}>0$ such that, if $U_{1}(F(\varphi)) \leqq \eta_{0}$, then $\|\varphi\|_{E} \leqq$ $K_{0}\left\|\left(I^{\omega}\right)^{\prime}(\varphi)\right\|_{E^{\prime}}$

Proof. Combining (H8) and Proposition 3.5, we get

$$
\begin{aligned}
\|\varphi\|_{E} \leqq & K\left(\left[\delta+C_{\delta} U_{1}(F(\varphi))^{\frac{1}{\beta}}\right]^{n_{0}}\|\varphi\|_{L^{2}}\right. \\
& \left.+\left[1+\delta+C_{\delta} U_{1}(F(\varphi))^{\frac{1}{\beta}}\right]^{n_{0}-1}\left\|\left(I^{\omega}\right)^{\prime}(\varphi)\right\|_{E^{\prime}}\right) \\
\leqq & \frac{\sqrt{m}\|\varphi\|_{L^{2}}}{2}+K^{\prime}\left\|\left(I^{\omega}\right)^{\prime}(\varphi)\right\|_{E^{\prime}} \leqq \frac{\|\varphi\|_{E}}{2}+K^{\prime}\left\|\left(I^{\omega}\right)^{\prime}(\varphi)\right\|_{E^{\prime}}
\end{aligned}
$$

for $\delta=\delta_{0}$ suitably chosen, and $U_{1}(F(\varphi)) \leqq \eta_{0}$. Hence the result. 


\section{Section 4. Existence of Solutions in the Symmetric Case}

We already explained in the introduction that existence and multiplicity results for localized solutions of the O.D.E. system

$$
\left\{\begin{aligned}
u^{\prime}+\frac{2 u}{r} & =v\left[g\left(v^{2}-u^{2}\right)-(m-\omega)\right] \\
v^{\prime} & =u\left[f\left(v^{2}-u^{2}\right)-(m-\omega)\right]
\end{aligned}\right.
$$

can be found in [5, 6, 8, 9 and 13]. The first result (see [9]) is the existence of one solution $(u, v)$ of $(1.9)$ satisfying $u(0)=0, v(0)>0$. Moreover $u, v$ decrease exponentially at infinity and $u, v>0$ in $\mathbb{R}^{+}$. Cazenave and Vazquez made a number of assumptions to prove it and in particular the nonlinearity $g$ had to be monotone nondecreasing. To our knowledge, the most general extension of their result is due to Merle:

Theorem 4.1. ([13]). Assume that $0<\omega<m$ and that $g \in C^{1}(\mathbb{R}, \mathbb{R})$ satisfies:

$$
\begin{aligned}
& g(0)=0, \\
& G(x) \geqq(m-\omega) x \quad \text { for } x \text { large }, \\
& G(x)=(m-\omega) x \Rightarrow g(x) \neq m-\omega .
\end{aligned}
$$

Then there exist functions $u, v$ which are global solution of (1.9) with

$$
u(0)=0, \quad v(0)>0,
$$

and for all $\theta \in(0, m-\omega)$, there exists $C_{\theta}>0$ s.t.

$$
0<u(r)<v(r) \leqq C_{\theta} e^{-\theta r} \text { for } r>0 .
$$

Remark. Remember that $G$ is defined by $G(t)=\int_{0}^{t} g(s) d s$.

The most general multiplicity result can be found in [5].

Theorem 4.2. ([5]). Assume that $0<\omega<m$ and assume that $g \in C^{1}(\mathbb{R})$ is a function satisfying

$$
\begin{aligned}
& g(0)=0, \\
& g \text { is monotone nondecreasing in }(0,+\infty), \\
& g(x)>m+\omega \text { for } x \text { large, } \\
& g^{\prime}\left(g^{-1}(m-\omega)\right)>0 .
\end{aligned}
$$

Then there exists a sequence of global solutions of (1.9), $\left(u_{n}, v_{n}\right)$ such that

$$
\begin{aligned}
& u_{n}(0)=0, \quad v_{n}(0)>0 \text { for all } n, \\
& \text { both } u_{n} \text { and } v_{n} \text { have } n \text { zeroes in }(0,+\infty), \\
& \left(u_{n}, v_{n}\right) \text { converges exponentially to }(0,0) \text { as } r \rightarrow+\infty
\end{aligned}
$$

In this paper we also prove existence and multiplicity results for (1.9). Our assumptions are different from those above, and somehow more restrictive. The main additional assumption we make is (H1), which says that $G$ is strictly superlinear. This is a usual assumption one encounters when using critical point theory.

Our result concerning the symmetric case is thus less general than Theorems 4.1 and 4.2 above. But our method is completely different: we use variational techniques. 
This provides us with additional information about the "energy" of the solutions and also estimates on their norm.

In order to begin our study about symmetric solutions of (1.5), i.e. solutions $\varphi$ of the form (1.8), we first write the expression of the energy functional for this particular class of functions.

If $\varphi \in E^{s}$, then

$$
\begin{aligned}
S\left(\begin{array}{l}
u \\
v
\end{array}\right)= & \frac{I^{\omega}(\varphi)}{4 \pi}=\int_{0}^{+\infty}\left[u^{\prime} v+\frac{2 u v}{r}-v^{\prime} u+m\left(v^{2}-u^{2}\right)-\right. \\
& \left.\omega\left(u^{2}+v^{2}\right)-G\left(v^{2}-u^{2}\right)\right] r^{2} d r
\end{aligned}
$$

and the Euler-Lagrange equation associated with the functional $S$ is the O.D.E. system (1.9).

Our main result concerning system (1.9) is Theorem 1. The remainder of this section is devoted to prove it.

In order to use variational techniques to solve (1.9) let us first use an approximation procedure.

For every $A \geqq A_{0}, A_{0}$ defined in (H3), we consider a nonlinearity $G_{A}$ which satisfies what follows (denoting $g_{A}=G_{A}^{\prime}$ ):

$$
\begin{aligned}
& G_{A}=\left\{\begin{array}{l}
G(x), \quad|x| \leqq A \\
G(A+1)(A+1)^{-\eta}|x|^{\eta}, \quad|x| \geqq A+1,
\end{array}\right. \\
& G_{A} \in C^{2}(\mathbb{R}), \quad G_{A}^{\prime} \geqq 0 \quad \forall x \in \mathbb{R} \\
& G_{A} \text { satisfies (H1) with } g=g_{A} \text { and } \theta \text { replaced by } \eta \text {, } \\
& \text { where } \eta=\min \left\{\theta, \frac{5}{4}\right\}
\end{aligned}
$$

Notice that (H1) and (H3) imply that for $A^{\prime}>A+1, G_{A^{\prime}} \geqq G_{A}$ on $\mathbb{R}$.

Then for $\varepsilon \geqq 0$ we define the functional $S_{A, \varepsilon}$ :

$$
\begin{aligned}
S_{A, \varepsilon}\left(\begin{array}{l}
u \\
v
\end{array}\right)= & \int_{0}^{+\infty}\left[u^{\prime} v+\frac{2 u v}{r}-v^{\prime} u+m\left(v^{2}-u^{2}\right)\right. \\
& \left.-\omega\left(u^{2}+v^{2}\right)-G_{A}\left(v^{2}-u^{2}\right)-\frac{4 \varepsilon}{5}\left(u^{2}+v^{2}\right)^{5 / 4}\right] r^{2} d r
\end{aligned}
$$

Note that the Euler-Lagrange equations corresponding to $S_{A, \varepsilon}^{\prime}\left(\begin{array}{l}u \\ v\end{array}\right)=0$ are

$$
\left\{\begin{aligned}
u^{\prime}+\frac{2 u}{r} & =v\left[g_{A}\left(v^{2}-u^{2}\right)+\varepsilon\left(u^{2}+v^{2}\right)^{1 / 4}-(m-\omega)\right] \\
v^{\prime} & =u\left[g_{A}\left(v^{2}-u^{2}\right)+\varepsilon\left(u^{2}+v^{2}\right)^{1 / 4}-(m+\omega)\right] .
\end{aligned}\right.
$$

Our strategy to prove existence and multiplicity of solutions to (1.9) consists in looking for critical points of $S_{A, \varepsilon}$, and passing to the limit as $\varepsilon$ goes to 0 . Finally, the critical points of $S_{A, 0}$ obtained by the above limiting procedure are shown to be solutions of (1.9) for $A$ large enough. 
Theorem 4.3. Under the assumptions of Theorem 1, for every $A \geqq A_{0}, \varepsilon>0$, the functional $S_{A, \varepsilon}$ satisfies the Palais-Smale condition, and there exists a sequence of distinct critical points of $S_{A, \varepsilon},\left(\begin{array}{c}u_{A, \varepsilon}^{n} \\ v_{A, \varepsilon}^{n}\end{array}\right)$ such that

$$
\rho_{A} \leqq c_{A, \varepsilon}^{n}=S_{A, \varepsilon}\left(\begin{array}{c}
u_{A, \varepsilon}^{n} \\
v_{A, \varepsilon}^{n}
\end{array}\right) \leqq c^{n}
$$

with $c^{n}$ independent of $\left(A \geqq A_{0}, \varepsilon>0\right), \rho_{A}>0$ independent of $\varepsilon \in\left(0, \varepsilon_{0}\right]$, and $c_{A, \varepsilon}^{n} \leqq c_{A, \varepsilon}^{n^{\prime}}$ for all $n^{\prime} \geqq n$. Moreover

$$
\lim _{n \rightarrow+\infty} c_{A, \varepsilon}^{n}=+\infty \text { for all } A, \varepsilon .
$$

Proof. Let $\left\{\left(\begin{array}{l}u_{j} \\ v_{j}\end{array}\right)\right\}$ be a sequence in $E^{s}$ such that

$$
\begin{aligned}
& S_{A, \varepsilon}\left(\begin{array}{c}
u_{j} \\
v_{j}
\end{array}\right) \underset{j \rightarrow+\infty}{\rightarrow} c, \\
& S_{A, \varepsilon}^{\prime}\left(\begin{array}{c}
u_{j} \\
v_{j}
\end{array}\right) \underset{j \rightarrow+\infty}{\rightarrow} 0, \text { in } E^{\prime} .
\end{aligned}
$$

From $(\mathrm{H} 1)$,

$$
\begin{aligned}
\left\|u_{j}^{2}+v_{j}^{2}\right\|_{L^{5 / 4}\left(r^{2} d r\right)}^{5 / 4} & \leqq \int_{0}^{+\infty} G_{A}\left(v_{j}^{2}-u_{j}^{2}\right)+\left(u_{j}^{2}+v_{j}^{2}\right)^{5 / 4} r^{2} d r \\
& \leqq \max \left\{\frac{5}{\varepsilon}, \frac{1}{\eta-1}\right\}\left(S_{A, \varepsilon}\left(\begin{array}{c}
u_{j} \\
v_{j}
\end{array}\right)-\frac{1}{2}<S_{A, \varepsilon}^{\prime}\left(\begin{array}{c}
u_{j} \\
v_{j}
\end{array}\right),\left(\begin{array}{c}
u_{j} \\
v_{j}
\end{array}\right)>\right) \\
& \leqq C_{1}+o\left(\left\|\varphi_{j}\right\|_{E}\right)
\end{aligned}
$$

for $j$ large enough, where $C_{1}$ depends only on $\varepsilon$ and $\varphi_{j}$ is defined by (1.7). By the definition of $g_{A}$ and (H2) we have

$$
\left.\left.\left|g_{A}(\bar{\varphi} \varphi) \varphi+\varepsilon\right| \varphi\right|^{1 / 2} \varphi|\leqq C| \varphi\right|^{3 / 2} \text { for all } \varphi \text {. }
$$

Therefore by the regularizing properties of the inverse of the linear Dirac operator,

$$
\left\|\varphi_{j}\right\|_{W^{1,5 / 3}\left(\mathbb{R}^{3}\right)+H^{1 / 2}\left(\mathbb{R}^{3}\right)} \leqq C_{2}+o\left(1+\left\|\varphi_{J}\right\|_{E}\right),
$$

and by the Sobolev embedding,

$$
\left\|\varphi_{j}\right\|_{E} \leqq C_{3} .
$$

Next, the embedding of $E^{s}$ in $L^{q}\left(\mathbb{R}^{3}\right)$ is compact for $2<q<3$. Indeed, this can be easily derived from the definition of $H^{1 / 2}$ as interpolation space between $H^{1}$ and $L^{2}$ and also from the fact that $X=\left\{u \in H^{1}\left(\mathbb{R}^{3}\right)\right.$; $\mathbf{u}$ is radially symmetric $\}$ is compactly embedded in $L^{q}\left(\mathbb{R}^{3}\right)$ for $2<q<6$ (see [18 or 2] for a proof of it). that

Hence, from $\left\{\varphi_{j}\right\}$ we can extract a subsequence, still denoted by $\left\{\varphi_{j}\right\}$, such

$$
\varphi_{j} \rightarrow \varphi_{*} \quad \text { in } L^{q}\left(\mathbb{R}^{3}\right), 2<q<3
$$


for some function $\varphi_{*}$ in $E^{s}$. By our assumptions on $g$ and $g_{A}$, we can then pass to the limit in (4.19) to infer that $S^{\prime}\left(\begin{array}{l}u_{*} \\ v_{*}\end{array}\right)=0$, where $\left(\begin{array}{l}u_{*} \\ v_{*}\end{array}\right)$ are associated to $\varphi_{*}$ by (1.8). Moreover (4.20) and the regularizing properties of $L_{D}^{-1}$ imply that

$$
\varphi_{j} \rightarrow \varphi_{*} \quad \text { in } H^{1 / 2}\left(\mathbb{R}^{3}\right),
$$

and therefore, from (4.19),

$$
S_{A, \varepsilon}\left(\begin{array}{l}
u_{*} \\
v_{*}
\end{array}\right)=c .
$$

So $S_{A, \varepsilon}$ satisfies the Palais-Smale condition.

For every $A \geqq A_{0}, \varepsilon>0$, the functional $S_{A, \varepsilon}$ satisfies all the properties necessary for the application of Corollary 2.4. Therefore there exists a sequence of positive min-max levels $\left(c_{A, \varepsilon}^{n}\right)_{n \geqq 0}$ such that $c_{A, \varepsilon}^{n} \leqq c_{A, \varepsilon}^{n^{\prime}} \forall n^{\prime} \geqq n$. The existence of $\rho_{A}, c^{n}$ is also given by Corollary 2.4. Moreover for every $n, A, \varepsilon$, the same corollary shows the existence of a sequence $\left\{\left(\begin{array}{l}u_{J} \\ v_{J}\end{array}\right)\right\}$ satisfying (4.19) with $c=c_{A, \varepsilon}^{n}$. So, by the Palais-Smale condition, there is a critical point at each level $c=c_{A, \varepsilon}^{n}$. The fact that $\lim _{n \rightarrow+\infty} c_{A, \varepsilon}^{n}=+\infty$ is again a consequence of the Palais-Smale condition, combined with classical properties of the genus (see e.g. [1]).

Proposition 4.4. Under the assumptions of Theorem 4.3 and if $\left(\begin{array}{l}u \\ v\end{array}\right)$ is any solution of $S_{A, \varepsilon}^{\prime}\left(\begin{array}{l}u \\ v\end{array}\right)=0$ with $c_{1} \leqq S_{A, \varepsilon}\left(\begin{array}{l}u \\ v\end{array}\right) \leqq c_{2}$ we have

$$
\begin{gathered}
\left|\int_{0}^{+\infty}\left(u^{\prime} v+\frac{2 u v}{r}-v^{\prime} u\right) r^{2} d r\right| \leqq M, \\
\left|\int_{0}^{+\infty}\left(m\left(v^{2}-u^{2}\right)-\omega\left(v^{2}+u^{2}\right)\right) r^{2} d r\right| \leqq M, \\
0 \leqq \int_{0}^{+\infty}\left(G_{A}\left(v^{2}-u^{2}\right)+\frac{4 \varepsilon}{5}\left(v^{2}+u^{2}\right)^{5 / 4}\right) r^{2} d r \leqq M,
\end{gathered}
$$

where $M$ is a constant which depends only on $c_{1}$ and $c_{2}$. This result is also valid for $\varepsilon=0$.

Proof. Let $\varphi$ satisfy (1.8). Then the function $F(\varphi)=G_{A}(\bar{\varphi} \varphi)+(4 \varepsilon / 5)|\varphi|^{5 / 2}$ satisfies all the assumptions of Proposition 3.3. Hence (3.8)-(3.10) hold. Moreover all the functions $G_{A}$ satisfy assumption (H7) with a uniform constant $\alpha=2 \eta>2$. Therefore, by Proposition 3.3 we infer that $M$ is independent of $\varepsilon$ and $A$.

Next we will be interested in obtaining some kind of a priori estimates for the critical points of $S_{A, \varepsilon}$, independently of $\varepsilon$ small enough and $A \geqq A_{0}$. We first obtain a uniform decay estimate at infinity.

Proposition 4.5. Let $c_{1}, c_{2} \in \mathbb{R}, \varepsilon_{0}>0$ be fixed. Then for all $\delta>0$, there exists $R>0$ such that for all $\varepsilon \in\left[0, \varepsilon_{0}\right], A \geqq A_{0}$,

$$
|u(r)|^{2}+|v(r)|^{2} \leqq \delta^{2} \quad \forall r \geqq R
$$

for all $\left(\begin{array}{c}u \\ v\end{array}\right) \in E^{s}$ such that $S_{A, \varepsilon}^{\prime}\left(\begin{array}{c}u \\ v\end{array}\right)=0, c_{1} \leqq S_{A, \varepsilon}\left(\begin{array}{c}u \\ v\end{array}\right) \leqq c_{2}$. 


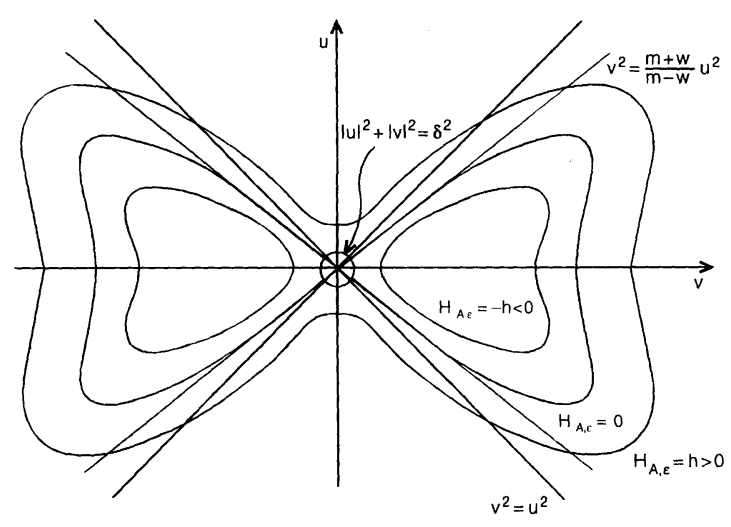

Fig. 1.

Proof. From Proposition 3.2, we know that $|u(r)|+|v(r)| \rightarrow 0$ as $r \rightarrow \infty$, and we want to find a uniform estimate on the convergence speed.

Take $\delta>0$, and assume by contradiction that there are sequences $r_{J} \rightarrow+\infty$, $\varepsilon_{j} \rightarrow \bar{\varepsilon} \in\left[0, \varepsilon_{0}\right], A_{j} \geqq A_{0}$ and $\left(\begin{array}{l}u_{j} \\ v_{j}\end{array}\right) \in E^{s}$ such that

$$
\begin{gathered}
\left|u_{j}\left(r_{j}\right)\right|^{2}+\left|v_{j}\left(r_{j}\right)\right|^{2}=\delta^{2} \quad \forall j \\
S_{A_{j}, \varepsilon_{j}}^{\prime}\left(\begin{array}{c}
u_{j} \\
v_{j}
\end{array}\right)=0 \quad \text { and } \quad c_{1} \leqq S_{A_{j}, \varepsilon_{j}}\left(\begin{array}{c}
u_{j} \\
v_{j}
\end{array}\right) \leqq c_{2} .
\end{gathered}
$$

Now let us compare the functions $u_{j}, v_{j}$ with the solutions of the Hamiltonian system

$$
\left\{\begin{array}{l}
u^{\prime}=v\left[g_{A_{j}}\left(v^{2}-u^{2}\right)+\varepsilon_{j}\left(u^{2}+v^{2}\right)^{1 / 4}-(m-\omega)\right] \\
v^{\prime}=u\left[g_{A_{j}}\left(v^{2}-u^{2}\right)+\varepsilon_{j}\left(u^{2}+v^{2}\right)^{1 / 4}-(m+\omega)\right]
\end{array}\right.
$$

The corresponding Hamiltonian is the function

$$
H_{A_{j}, \varepsilon_{j}}(u, v)=\frac{1}{2}\left[G_{A_{j}}\left(v^{2}-u^{2}\right)+\frac{4 \varepsilon_{j}}{5}\left(u^{2}+v^{2}\right)^{5 / 4}-m\left(v^{2}-u^{2}\right)+\omega\left(u^{2}+v^{2}\right)\right]
$$

for $(u, v) \in \mathbb{R}^{2}$.

From $(\mathrm{H} 4),(0,0)$ is a hyperbolic equilibrium for $(4.27)$. From $(\mathrm{H} 1,3)$, the zero energy level $\left\{H_{A_{J}, \varepsilon_{J}}=0\right\}$ is compact. Moreover, from $(\mathrm{H} 3), H_{A_{j}, \varepsilon_{j}}^{\prime} \cdot(u, v)-$ $2 H_{A_{j}, \varepsilon_{j}} \geqq(\eta-1)\left(G_{A_{j}}\left(v^{2}-u^{2}\right)+\frac{4 \varepsilon_{j}}{5}\left(u^{2}+v^{2}\right)^{5 / 4}\right)>0$, so that there is no other equilibrium point than $(0,0)$ in the domain $\left\{-h \leqq H_{A_{j}, \varepsilon_{j}} \leqq h\right\}$ for $h>0$ small enough. It is not difficult to see that $h$ can be chosen independently of $j$.

Studying the flow of (4.27) in the domain $\left\{-h \leqq H_{A_{J}, \varepsilon_{J}} \leqq h\right\}$ (see Figure 1), one easily obtains the following property:

For $\delta$ chosen small enough, there are $D, L, \sigma>0$ independent of $j$ (but depending on $\delta$ ), such that if $\left(\bar{u}_{j}, \bar{v}_{J}\right)$ is the solution of the Hamiltonian system (4.27) 
satisfying $\bar{u}_{j}\left(r_{j}\right)=u_{j}\left(r_{j}\right), \bar{v}_{j}\left(r_{j}\right)=v_{j}\left(r_{j}\right)$, then there exists $\bar{r}_{j}$ such that $\left|\bar{r}_{j}-r_{j}\right| \leqq D$ and

$$
G\left(\bar{v}_{j}^{2}(r)-\bar{u}_{j}^{2}(r)\right)>2 \sigma \text { for all } r \in\left[\bar{r}_{J}-L, \bar{r}_{J}+L\right] .
$$

Now, for $r_{j}$ very large, the solutions $\left(u_{j}, v_{j}\right)$ and $\left(\bar{u}_{j}, \bar{v}_{j}\right)$ are very close on $\left[r_{j}-D-L, r_{j}+D+L\right]$. A precise proof of this property is given in [8], Lemma 2.5. Therefore,

$$
G\left(v_{j}^{2}(r)-u_{j}^{2}(r)\right)>\sigma
$$

for $r \in\left[\bar{r}_{j}-L, \bar{r}_{j}+L\right]$ and $j$ large enough. But this contradicts (4.24) because of assumption (H3). The proof is complete.

The uniform asymptotic decay obtained above can be shown to be exponential:

Proposition 4.6. Let $\varepsilon_{0}>0$ be fixed. Then for every $c_{1}, c_{2} \in \mathbb{R}$, there exist constants $R, K, \sigma>0$ such that

$$
|u(r)|+|v(r)| \leqq K e^{-\sigma r}, \quad \forall r \geqq R
$$

for all $\varepsilon \in\left[0, \varepsilon_{0}\right], A \geqq A_{0}$ and for any critical point $\left(\begin{array}{l}u \\ v\end{array}\right)$ of $S_{A, \varepsilon}$ such that $c_{1} \leqq S_{A, \varepsilon}\left(\begin{array}{c}u \\ v\end{array}\right) \leqq c_{2}$.

Proof. Taking derivatives in (4.16) and after tedious computations we find the following:

$$
\begin{cases}-v^{\prime \prime}+\left(m^{2}-\omega^{2}\right) v=o(v)+o\left(v^{\prime}\right) & \text { for } r \text { large, } \\ -u^{\prime \prime}+\left(m^{2}-\omega^{2}\right) u=o(u)+o\left(u^{\prime}\right) & \text { for } r \text { large. }\end{cases}
$$

We conclude by an easy application of the maximum principle.

Remark. Both the uniform decay and the exponential decay properties we proved above are independent of $\varepsilon \in\left[0, \varepsilon_{0}\right]$ and also of $A \geqq A_{0}$. The main reason for this is that the constant $M$ in (4.24) is independent of $\varepsilon$ and $A$.

Now we have all the ingredients which are necessary to pass to the limit as $\varepsilon$ goes to 0 . We begin by deriving some a priori estimates.

Proposition 4.7. For all $c_{1}, c_{2} \in \mathbb{R}$ and $\varepsilon_{0}>0$, there exists a constant $C>0$ such that for all $\varepsilon \in\left(0, \varepsilon_{0}\right], A \geqq A_{0},\left(\begin{array}{l}u \\ v\end{array}\right)$ critical point of $S_{A, \varepsilon}$ satisfying $c_{1} \leqq S_{A, \varepsilon}\left(\begin{array}{l}u \\ v\end{array}\right) \leqq$ $c_{2}$ we have

$$
\|u\|_{L^{2}\left((0,+\infty) ; r^{2} d r\right)}+\|v\|_{L^{2}\left((0,+\infty) ; r^{2} d r\right)} \leqq C .
$$

Moreover, for every $p>1$ there exists $C_{p}(A)>0$ independent of $\varepsilon \in\left(0, \varepsilon_{0}\right]$, such that

$$
\|\varphi\|_{W^{1, p}\left(\mathbb{R}^{3}\right)} \leqq C_{p}(A), \quad \varphi \text { defined by }(1.8) .
$$

Proof. From Proposition 4.6, there are $R, \bar{C}>0$ such that

$$
\|u\|_{L^{2}\left((R,+\infty) ; r^{2} d r\right)}+\|v\|_{L^{2}\left((R,+\infty) ; r^{2} d r\right)} \leqq \bar{C} .
$$

Then $(\mathrm{H} 1,3),(4.24)$ and $G_{A}$ 's definition yield the existence of $\eta>1, A_{0}>0, M>$ 0 , independent of $\varepsilon \in\left[0, \varepsilon_{0}\right], A \geqq A_{0}$ and such that

$$
\int_{0}^{+\infty} r^{2} d r\left|v^{2}-u^{2}\right|^{\eta} 1_{\left\{\left|v^{2}-u^{2}\right| \geqq A_{0}\right\}} \leqq \frac{M}{G\left(A_{0}\right)}<\infty,
$$


hence

$$
\int_{0}^{R}\left|v^{2}-u^{2}\right| r^{2} d r \leqq \frac{R^{3} A_{0}}{3}+\frac{M A_{0}^{1-\eta}}{G\left(A_{0}\right)} .
$$

Then estimate (4.23) together with Proposition 4.6 proves (4.32). Finally (4.33) follows from (4.32) and Proposition 3.2.

We now can pass to the limit as $\varepsilon$ goes to 0 . The next step will be to obtain a priori estimates independent of $A$.

Proposition 4.8. Let $A \geqq A_{0}$ be fixed. Under the assumptions of Theorem 1 and (4.14), there exists an infinite sequence $\left(u_{A}^{n}, v_{A}^{n}\right)$ of distinct critical points of the functional $S_{A, 0}$, i.e. solutions of (1.5) with $g=g_{A}$. Moreover, for every $n \in \mathbb{N}$ there exist positive constants $C, K, \sigma, R$ independent of $A$ (but depending on $n$ ), such that

$$
\begin{gathered}
\left\|u_{A}^{n}\right\|_{L^{2}\left((0,+\infty) ; r^{2} d r\right)}+\left\|v_{A}^{n}\right\|_{L^{2}\left((0,+\infty) ; r^{2} d r\right)} \leqq C \\
\left|u_{A}^{n}(r)\right|+\left|v_{A}^{n}(r)\right| \leqq K e^{-\sigma r} \quad \forall r \geqq R
\end{gathered}
$$

Proof. The above series of lemmas and propositions provide us with the estimates which are needed to pass to the limit in (4.16). Indeed Theorem 4.3 yields the existence of an infinite sequence of distinct critical points of $S_{A, \varepsilon}$. Then we use Proposition 4.7 and the compact embedding $W_{\text {rad }}^{1,2} \subset L^{q}\left(\mathbb{R}^{3}\right), 2<q<6$, to pass to the limit. By doing this we obtain a nondecreasing sequence of positive critical levels $c_{A, 0}^{n}>0$ such that for every $n$, there is a nontrivial solution of (1.5), $\left(u_{A}^{n}, v_{A}^{n}\right)$, such that $S_{A, 0}\left(\begin{array}{c}u_{A}^{n} \\ v_{A}^{n}\end{array}\right)=c_{A, 0}^{n}$. The estimates (4.36) and (4.37) then follow from Propositions 4.6 and 4.7 .

If the sequence $c_{A, 0}^{n}$ is strictly increasing, the proof is complete. Assume therefore that there exists $\bar{n}$ such that

$$
c_{A, 0}^{\bar{n}}=c_{A, 0}^{\bar{n}+1}=c,
$$

and let us denote by $K_{c}$ the set of critical points of $S_{A, 0},\left(\begin{array}{l}u \\ v\end{array}\right)$, such that $S_{A, 0}\left(\begin{array}{l}u \\ v\end{array}\right)=c$. If $K_{c}$ is not compact, its cardinal is infinite, and the proof is over.

To treat the remaining possibilities, we need the following lemma:

Lemma 4.9. Let $A \geqq A_{0}$ be fixed. We assume that (4.38) holds and that $K_{c}$ is compact. Then:

(i) Let $\mathscr{U}, \mathscr{V}$ be two bounded open sets in $E^{s}$ such that $K_{c} \subset \mathscr{U} \subset \overline{\mathscr{U}} \subset \mathscr{V}$. Then there exist constants $\delta_{1}, \alpha, \varepsilon_{1}>0$ such that for all $\varepsilon \in\left(0, \varepsilon_{1}\right]$, for all $\left(\begin{array}{l}u \\ v\end{array}\right) \in(\mathscr{V} \backslash \mathscr{U}) \cap\left\{\left(\begin{array}{l}u \\ v\end{array}\right) ; S_{A, \varepsilon}\left(\begin{array}{l}u \\ v\end{array}\right) \in\left[c-\delta_{1}, c+\delta_{1}\right]\right\}$ we have

$$
\left\|S_{A, \varepsilon}^{\prime}\left(\begin{array}{l}
u \\
v
\end{array}\right)\right\|_{E^{\prime}} \geqq \alpha
$$

(ii) For all $\varepsilon \in\left(0, \varepsilon_{1}\right]$, if $\left(\begin{array}{l}u \\ v\end{array}\right) \notin \mathscr{U}$ and if $S_{A, \varepsilon}\left(\begin{array}{l}u \\ v\end{array}\right) \in\left[c-\delta_{1}, c+\delta_{1}\right]$, then

$$
\left\|S_{A, \varepsilon}^{\prime}\left(\begin{array}{l}
u \\
v
\end{array}\right)\right\| \geqq \beta(\varepsilon)>0 .
$$

This lemma has the following consequence (whose proof is standard and will be omitted): 
Corollary 4.10. Under the above assumptions, there exists $\delta_{2}>0$ such that for any $0<\varepsilon \leqq \varepsilon_{1}$ there is a time $T(\varepsilon)>0$ with

$$
S_{A, \varepsilon}\left(h_{\varepsilon}\left(T(\varepsilon),\left(\begin{array}{l}
u \\
v
\end{array}\right)\right)\right) \leqq c-\delta_{2}
$$

for all $\left(\begin{array}{l}u \\ v\end{array}\right) \notin y$ and such that $S_{A,:}\left(\begin{array}{l}u \\ v\end{array}\right) \leqq c+\delta_{2}$, where by $h_{i}$, we denote the flow induced by $-\nabla S_{A, \varepsilon}=-|D|^{-1} S_{A, \varepsilon}^{\prime}$.

Proof of Lemma 4.9. $\mathscr{V}$ is bounded in $E^{s}$, and $E^{s}$ is compactly embedded in $L^{q}$ for $2 \leqq q<3$. As a consequence, $S_{A, 0}$ restricted to the domain $\mathscr{V} \backslash \mathscr{U}$ satisfies the Palais-Smale condition, and (i) follows from that.

From Proposition 4.7 and the same compact embedding as above, for $\varepsilon>0$ small enough, any critical point $(u, v)$ of $S_{A, \varepsilon}$ such that $S_{A, \varepsilon}(u, v) \in\left[c-\delta_{0}, c+\delta_{0}\right]$ must be in $\mathscr{U}$. But from Theorem 4.3, we know that $S_{A, \imath}$ satisfies the Palais-Smale condition, hence (ii).

Let us come back to the proof of Proposition 4.8. We make the same assumptions as in Lemma 4.9. Let us take two bounded open neighborhoods of $K_{c}, \mathscr{U}, \mathscr{V}$ such that $\gamma\left(K_{c}\right)=\gamma(\mathscr{V})$ and $K_{c} \subset \mathscr{U} \subset \overline{\mathscr{U}} \subset \mathscr{V}, \gamma$ being the genus. By Corollary 4.10,

$$
\begin{aligned}
d_{\varepsilon} & =\gamma\left(h_{\varepsilon}\left(T(\varepsilon),\left\{\left(\begin{array}{l}
u \\
v
\end{array}\right) ; S_{A, \varepsilon}\left(\begin{array}{l}
u \\
v
\end{array}\right) \leqq c+\delta_{2}\right\} \backslash \mathscr{y}\right)\right) \\
& \leqq l_{\varepsilon}=\gamma\left(\left\{\left(\begin{array}{l}
u \\
v
\end{array}\right) ; S_{A, \varepsilon}\left(\begin{array}{l}
u \\
v
\end{array}\right) \leqq c-\delta_{2}\right\}\right) \leqq \bar{n}-2 .
\end{aligned}
$$

But we also have $d_{\varepsilon}+\gamma(\mathscr{V}) \geqq \bar{n}$. Therefore, $\gamma\left(K_{c}\right) \geqq 2$, so $K_{c}$ is an infinite set.

Remark 4.11. Notice that Propositions 3.2 and 4.8 easily imply that $\left(u_{A}^{n}, v_{A}^{n}\right) \in$ $L^{\infty}\left(\mathbb{R}^{+}\right)^{2}$ for all $n, A \geqq A_{0}$. Moreover from it we infer that $u_{A}(0)=0 \quad \forall n, A \geqq$ $A_{0}$. Our goal now is to prove that for every $n$ these solutions are uniformly bounded in $\left(L^{\infty}\right)^{2}$, independently of $A$. Of course, that will show that for $A$ large enough, $\left(u_{A}^{n}, v_{A}^{n}\right)$ is a solution of system (1.9).

Lemma 4.12. Let $n \in \mathbb{N}$ and $\left(u_{A}^{n}, v_{A}^{n}\right)$ as in Proposition 4.8. Then there exists $C_{1}>0$ depending on $n$ but independent of $A \geqq A_{0}$, such that

$$
\left|v_{A}^{n}(0)\right| \leqq C_{1} \text {. }
$$

Proof. This easily follows from Lemma 2.9 in [8]. Indeed this result shows that $\left(v_{A}^{n}\right)^{2}(r)-\left(u_{A}^{n}\right)^{2}(r) \geqq e^{-4}\left(v_{A}^{n}(0)\right)^{2}$ for all $r \in[0,1 /()]$. This and (4.36) allow us to conclude.

Proposition 4.13. For every $n \in \mathbb{N}$ there exists $C_{2}>0$ depending on $n$ but independent of $A \geqq A_{0}$, such that

$$
\left\|u_{A}^{n}\right\|_{L^{\infty}\left(\mathbb{R}^{+}\right)}+\left\|v_{A}^{n}\right\|_{L^{\infty}\left(\mathbb{R}^{+}\right)} \leqq C_{2} .
$$

Corollary 4.14. For every $n \in \mathbb{N}$ there exists $A(n) \geqq A_{0}$ such that for all $A \geqq$ $A(n),\left(u_{A}^{n}, v_{A}^{n}\right)$ is a solution of (1.9).

Remark. Of course this proves Theorem 1.

Proof of Proposition 4.13. Let $n \in \mathbb{N}$ be fixed. 
As in [8] we see that for all $A \geqq A_{0}$,

$$
\frac{d}{d r}\left(\left(v_{A}^{n}\right)^{2}(r)-\left(u_{A}^{n}\right)^{2}(r)\right)=\frac{4}{r}\left(u_{A}^{n}\right)^{2}(r)-4 \omega u_{A}^{n}(r) v_{A}^{n}(r) .
$$

Since by Proposition $4.7, u_{A}^{n}$ and $v_{A}^{n}$ are bounded in $L^{2}\left(0,+\infty ; r^{2} d r\right)$ uniformly in $A \geqq A_{0}$, (4.42) implies that for every $\rho>0,\left(\left(v_{A}^{n}\right)^{2}-\left(u_{A}^{n}\right)^{2}\right)$ is bounded in $L^{\infty}(\rho,+\infty)$ independently of $A \geqq 1$. Moreover $\left(u_{A}^{n}, v_{A}^{n}\right)$ is a solution of the system

$$
\left\{\begin{array}{l}
u_{A}^{n}(r)=-\frac{1}{r^{2}} \int_{r}^{+\infty} s^{2} v_{A}^{n}(s)\left[g_{A}\left(\left(v_{A}^{n}\right)^{2}-\left(u_{A}^{n}\right)^{2}\right)-(m-\omega)\right] d s \\
v_{A}^{n}(r)=-\int_{r}^{+\infty} u_{A}^{n}(s)\left[g_{A}\left(\left(v_{A}^{n}\right)^{2}-\left(u_{A}^{n}\right)^{2}\right)-(m+\omega)\right] d s
\end{array}\right.
$$

Hence the above estimates and Proposition 4.7 imply that for any $\rho>0, u_{A}^{n}$ and $v_{A}^{n}$ are bounded in $L^{\infty}(\rho,+\infty)$ independently of $A \geqq 1$. Indeed the uniform exponential decay at infinity implies that $v_{A}^{n}$ and $u_{A}^{n}$ are uniformly bounded in $L^{1}\left([\rho,+\infty) ; r^{2} d r\right)$.

But lemma 4.12 and the definition of $g_{A}$ enable us to use a contraction theorem in order to prove local existence of bounded solutions of (4.16) (with $\varepsilon=0$ ) in an interval $[0, \delta], \delta>0$, with the initial values $\left(0, v_{A}^{n}(0)\right)$. Moreover $\delta$ is independent of $A \geqq A_{0}$, as well as the estimate on $\left|u_{A}^{n}(r)\right|+\left|v_{A}^{n}(r)\right|$ for $r \in[0, \delta]$. Therefore, if we choose $\rho=\delta$, the proof of Proposition 4.13 (and also of Theorem 1, by the remark above) is complete.

\section{Section 5. Proof of Theorems 2 and 3}

We start with the following result, which describes the behavior of bounded critical sequences:

Lemma 5.1. Assume that $F$ satisfies (H5, 6, 7, 8), and take $\omega \in(0, m)$.

There is $\lambda_{0}>0$ such that any non-zero $\varphi \in E$, solution of (1.5), satisfies $I^{\omega}(\varphi) \geqq \lambda_{0}$.

Moreover, if $\left(\varphi_{n}\right)_{n \geqq 0}$ is a sequence in $E$ such that $\left\|\varphi_{n}\right\|_{E}$ is bounded independently of $n$, and $\left(I^{\omega}\right)^{\prime}\left(\varphi_{n}\right) \rightarrow 0$ in $E^{\prime}, 0<c_{1} \leqq I^{\omega}\left(\varphi_{n}\right) \leqq c_{2}<+\infty$, then there are $p$ non-zero solutions $\phi^{1}, \ldots, \phi^{p}$ of $(1.5)$ in $E$, and $p$ sequences $\left(X_{n}^{1}\right)_{n \geqq 0} \cdots$ $\left(X_{n}^{p}\right)_{n \geqq 0}, X_{n}^{i} \in \mathbb{R}^{3}$, such that $i \neq j \Rightarrow\left|X_{n}^{i}-X_{n}^{j}\right| \rightarrow_{n \rightarrow \infty} \infty$, and, after extraction,

$$
\left\|\varphi_{n}-\sum_{i=1}^{p} \phi^{i}\left(\cdot-X_{n}^{i}\right)\right\|_{E} \underset{n \rightarrow \infty}{\rightarrow} 0 .
$$

Each $\phi^{l}$ satisfies $I^{\omega}\left(\phi^{i}\right) \in\left[\lambda_{0}, c_{2}\right]$, and $1 \leqq p \leqq \frac{c_{2}}{\lambda_{0}}$.

Proof. The existence of $\lambda_{0}$ follows from (H7), which gives

$$
\|F(\varphi)\|_{L^{1}} \leqq \frac{2}{\alpha-2} I^{\omega}(\varphi)
$$

and from Corollary 3.6. 
The existence of $\phi^{1}, \ldots, \phi^{p}$ can be proved thanks to classical arguments of the concentration-compactness theory (see [12]): "vanishing" is forbidden by Corollary 3.6.

The estimates on $I^{\omega}\left(\phi^{i}\right)$ and $p$ come from the inequality $\sum_{l=1}^{p} I^{\omega}\left(\phi^{l}\right) \leqq c_{2}$.

The main problem we have to face is to find an estimate on the E-norm of appropriate critical sequences.

As in Sect. 4, we need auxiliary functionals. Assuming that $F$ satisfies (H5, 6, 7, $8,9)$, we define, for $\varepsilon>0, \varphi \in \mathbb{C}^{4}, F_{\varepsilon}(\varphi)=F(\varphi)+\varepsilon|\varphi|^{\alpha_{2}}$, where $\alpha_{2}$ is the constant of (H5).

Clearly, $F_{\varepsilon}$ satisfies $(\mathrm{H} 5,6,7,8,9)$. We also define

$$
\begin{aligned}
I_{\varepsilon}^{\omega}(\varphi) & =I^{\omega}(\varphi)-\varepsilon \int_{\mathbb{R}^{3}}|\varphi|^{\alpha_{2}} \\
& =\frac{1}{2}\langle\varphi, D \varphi\rangle-\frac{\omega}{2}\|\varphi\|_{L^{2}}^{2}-\int_{\mathbb{R}^{3}} F_{\varepsilon}(\varphi) .
\end{aligned}
$$

For $\varepsilon$ fixed, we associate to $I_{\varepsilon}^{\omega}$ a positive min-max level $c_{\varepsilon}(\omega)$ and a sequence $\left(\varphi_{\varepsilon, n}\right)_{n \geqq 0}$ in $E$, such that $I_{\varepsilon}^{\omega}\left(\varphi_{\varepsilon, n}\right) \rightarrow_{n \rightarrow \infty} c_{\varepsilon}(\omega)$ and

$$
\left\|\left(I_{\varepsilon}^{\omega}\right)^{\prime}\left(\varphi_{\varepsilon, n}\right)\right\|_{E^{\prime}} \underset{n \rightarrow \infty}{\rightarrow} 0
$$

thanks to Corollary 2.6. There is also a level $c(\omega)$ corresponding to $I^{\omega}$, and $c_{\varepsilon}(\omega) \rightarrow_{n \rightarrow \infty} c(\omega)$.

We have the following result

Lemma 5.2. Assume that $F$ satisfies (H5), (H6),(H7), (H8), (H9), and consider $I_{\varepsilon}^{\omega}, c_{\varepsilon}(\omega)$ defined as above.

Then there is $\varphi_{\varepsilon} \in E$ with $\left(I_{\varepsilon}^{\omega}\right)^{\prime}\left(\varphi_{\varepsilon}\right)=0$ and

$$
0<\frac{\lambda_{0}}{2} \leqq I_{\varepsilon}^{\omega}\left(\varphi_{\varepsilon}\right) \leqq c_{\varepsilon}(\omega) \quad \text { for } \varepsilon \text { small enough }
$$

where $\lambda_{0}$ comes from Lemma 5.1 .

Proof. Let $\varepsilon>0$ be fixed and $\left\{\varphi_{n}\right\} \subset E$ be such that

$$
\left(I_{\varepsilon}^{\omega}\right)^{\prime}\left(\varphi_{n}\right) \rightarrow 0 \text { and } I_{\varepsilon}^{\omega}\left(\varphi_{n}\right) \rightarrow c_{\varepsilon}(\omega) .
$$

First we prove that $\left\|\varphi_{n}\right\|_{E} \leqq C$, where $C$ is independent of $n$. We do this as in the proof of Theorem 4.3. Then we apply Lemma 5.1 to $\left\{\varphi_{n}\right\}$. This yields the existence of at least one critical point of $I_{\varepsilon}^{\omega}, \varphi_{\varepsilon}$, such that

$$
0<\lambda_{0}(\varepsilon) \leqq I_{\varepsilon}^{\omega}\left(\varphi_{\varepsilon}\right) \leqq c_{\varepsilon}(\omega)
$$

and

$$
0<\frac{\lambda_{0}}{2} \leqq \lambda_{0}(\varepsilon)
$$

for $\varepsilon$ small enough.

Lemma 5.3. Assume that $F$ satisfies $(\mathrm{H} 5,6,7,8,9)$, and consider the functions $\varphi_{\varepsilon}$ found in Lemma 5.2 .

Then for $\varepsilon_{1}$ small enough $\left\|\varphi_{\varepsilon}\right\|_{L^{2}\left(\mathbb{R}^{3}, \mathbb{C}^{4}\right)}$ is bounded independently of $\varepsilon \in\left(0, \varepsilon_{1}\right]$. 
Proof. We take $\varepsilon_{1}$ such that $F_{\varepsilon}$ satisfies $(\mathrm{H} 5,6,7,8,9)$ with constants $a_{i}, \alpha_{i}, \alpha, \beta, C_{\delta}, v$ independent of $\varepsilon \in\left(0, \varepsilon_{1}\right]$, and $\frac{\lambda_{0}}{2} \leqq I_{\varepsilon}^{\omega}\left(\varphi_{\varepsilon}\right) \leqq c_{\varepsilon}(\omega)$, as in Lemma 5.2, with moreover $c_{\varepsilon}(\omega) \leqq 2 c(\omega)$. From Proposition 3.3, each $\varphi_{\varepsilon}$ satisfies

$$
\begin{gathered}
\left.\left|m \int_{\mathbb{R}^{3}}\left(\varphi_{\varepsilon} \overline{\varphi_{\varepsilon}}\right)-\omega \int_{\mathbb{R}^{3}}\right| \varphi_{\varepsilon}\right|^{2} \mid \leqq K^{(0)}, \\
\int_{\mathbb{R}^{3}} F_{\varepsilon}\left(\varphi_{\varepsilon}\right) \leqq K^{(1)}, \quad K^{(1)} \text { independent of } \varepsilon .
\end{gathered}
$$

We take $v>0$, whose value will be chosen later. Recalling the notation $Q_{l}=\left\{\left(x^{1}, x^{2}, x^{3}\right), l^{l} \leqq x^{i} \leqq l^{i}+1(\forall i)\right\}, n \in \mathbf{Z}^{3}$, there is a finite set $L \subset \mathbf{Z}^{3}$, with

$$
0 \leqq \operatorname{Card}(L) \leqq \frac{K^{(1)}}{\eta^{\beta}},
$$

and such that

$$
\left(l \in \mathbf{Z}^{3} \backslash L \Rightarrow \int_{Q_{l}} F_{\varepsilon}\left(\varphi_{\varepsilon}\right) \leqq \eta^{\beta}\right) .
$$

We take $R>0$, to be chosen later, and we define

$$
V_{R}=\left\{x \in \mathbb{R}^{3} / \operatorname{dist}\left(x, \bigcup_{l \in L} \mathrm{Q}_{l}\right) \leqq R\right\} .
$$

There is a smooth function $\theta_{R}: \mathbb{R}^{3} \rightarrow[0,1]$, such that $\theta_{R} \equiv 0$ on $V_{0}, \theta_{R} \equiv 1$ on $\mathbb{R}^{3} \backslash V_{R}$, and $\left\|\nabla \theta_{R}\right\|_{L^{\infty}} \leqq \frac{K^{(2)}}{R}, K^{(2)}$ independent of $R$. We have

$$
D\left(\theta_{R} \varphi_{\varepsilon}\right)-\omega \theta_{R} \varphi_{\varepsilon}=\theta_{R} F_{\varepsilon}^{\prime}\left(\varphi_{\varepsilon}\right)+\Omega_{R, \varepsilon},
$$

with

$$
\left\|\Omega_{R, \varepsilon}\right\|_{E^{\prime}} \leqq \frac{1}{\sqrt{m}}\left\|\Omega_{R, \varepsilon}\right\|_{L^{2}} \leqq \frac{K^{(3)}}{R}\left\|\varphi_{\varepsilon}\right\|_{L^{2}}
$$

and, from (H8)

$$
\left|\theta_{R} F_{\varepsilon}^{\prime}\left(\varphi_{\varepsilon}\right)\right| \leqq\left(\delta+C_{\delta} F\left(\varphi_{\varepsilon}\right)^{\frac{1}{\beta}}\right)\left|\theta_{R} \varphi_{\varepsilon}\right|
$$

almost everywhere.

But, from (5.4), we have

$$
\begin{aligned}
& U_{\beta}\left(\left[\delta+C_{\delta} F\left(\varphi_{\varepsilon}\right)^{\frac{1}{\beta}}\right] \cdot 1_{\mathbb{R}^{3} \backslash \bigcup_{l \in L} \mathrm{Q}_{l}}\right) \\
& \leqq \delta+C_{\delta} U_{1}\left(F\left(\varphi_{\varepsilon}\right) 1_{\mathbb{R}^{3} \backslash \bigcup_{l \in L} \mathrm{Q}_{l}}\right)^{\frac{1}{\beta}} \leqq \delta+C_{\delta} \eta
\end{aligned}
$$


So, from Proposition 3.5,

$$
\begin{aligned}
\left\|\theta_{R} \varphi_{\varepsilon}\right\|_{L^{2}} & \leqq \frac{K}{\sqrt{m}}\left(\left[\delta+C_{\delta} \eta\right]^{n_{0}} \cdot\left\|\varphi_{\varepsilon}\right\|_{L^{2}}+\left[1+\delta+C_{\delta} \eta\right]^{n_{0}-1} \cdot\left\|\Omega_{R, \varepsilon}\right\|_{E^{\prime}}\right) \\
& \leqq \frac{K}{\sqrt{m}}\left[\left[\delta+C_{\delta} \eta\right]^{n_{0}}+\left[1+\delta+C_{\delta} \eta\right]^{n_{0}-1} \cdot \frac{K^{(3)}}{R}\right]\left\|\varphi_{\varepsilon}\right\|_{L^{2}} \\
& \leqq \sqrt{\frac{\omega}{2 m}}\left\|\varphi_{\varepsilon}\right\|_{L^{2}},
\end{aligned}
$$

for $\delta=\delta_{0}, \eta=\eta_{0}$ and $R=R_{0}$ suitably chosen. As a consequence,

$$
m\left|\int_{\mathbb{R}^{3} \backslash V_{R_{0}}}\left(\varphi_{\varepsilon} \overline{\varphi_{\varepsilon}}\right)\right| \leqq \frac{\omega}{2}\left\|\varphi_{\varepsilon}\right\|_{L^{2}\left(\mathbb{R}^{3}\right)}^{2} .
$$

Now, from (H9), (5.2), (5.3) and (5.5),

$$
\begin{aligned}
m\left|\int_{V_{R_{0}}}\left(\varphi_{\varepsilon} \overline{\varphi_{\varepsilon}}\right)\right| & \leqq m\left(\mathscr{V} o l\left(V_{R_{0}}\right)\right)^{\frac{v-1}{v}}\left(\int_{V_{R_{0}}}\left|\varphi_{\varepsilon} \overline{\varphi_{\varepsilon}}\right|^{v}\right)^{\frac{1}{v}} \\
& \leqq K^{(4)}\left(\int_{V_{R_{0}}} F\left(\varphi_{\varepsilon}\right)+1\right)^{\frac{1}{v}} \leqq K^{(5)}
\end{aligned}
$$

Combining (5.1) with (5.10) and (5.11), we get

$$
\begin{aligned}
\omega\left\|\varphi_{\varepsilon}\right\|_{L^{2}\left(\mathbb{R}^{3}\right)}^{2} & \leqq K^{(0)}+m \int_{\mathbb{R}^{3}}(\varphi \bar{\varphi}) \\
& \leqq K^{(0)}+\frac{\omega}{2}\left\|\varphi_{\varepsilon}\right\|_{L^{2}\left(\mathbb{R}^{3}\right)}^{2}+K^{(5)},
\end{aligned}
$$

and

$$
\left\|\varphi_{\varepsilon}\right\|_{L^{2}\left(\mathbb{R}^{3}\right)}^{2} \leqq \frac{2\left(K^{(0)}+K^{(5)}\right)}{\omega}=K^{(6)},
$$

which proves the lemma.

Combining Lemma 5.3 and Proposition 3.2, we find that $\varphi_{\varepsilon}$, solution of

$$
D \varphi_{\varepsilon}-\omega \varphi_{\varepsilon}=F_{\varepsilon}^{\prime}\left(\varphi_{\varepsilon}\right)=F^{\prime}\left(\varphi_{\varepsilon}\right)+\varepsilon \alpha_{2}\left|\varphi_{\varepsilon}\right|^{\alpha_{2}-2} \varphi_{\varepsilon}
$$

is bounded in $E$ (and even in any $W^{1, q}, q \in[2, \infty)$ ) independently of $\varepsilon \in\left(0, \varepsilon_{1}\right]$. So

$$
\begin{aligned}
\left\|\left|\varphi_{\varepsilon}\right|^{\alpha_{2}-2} \varphi_{\varepsilon}\right\|_{E^{\prime}} & =O\left(\left\|\left|\varphi_{\varepsilon}\right|^{\alpha_{2}-2} \varphi_{\varepsilon}\right\|_{L^{\frac{\alpha_{2}}{\alpha_{2}}}}\right) \\
& =O\left(\left\|\varphi_{\varepsilon}\right\|_{L^{\alpha_{2}}}^{\alpha_{2}-2}\right)=O(1)
\end{aligned}
$$

hence

$$
\left\|\left(I^{\omega}\right)^{\prime}\left(\varphi_{\varepsilon}\right)\right\|_{E^{\prime}} \rightarrow 0 \quad \text { as } \quad \varepsilon \rightarrow 0
$$


Moreover,

$$
0<\frac{\lambda_{0}}{2} \leqq I^{\omega}\left(\varphi_{\varepsilon}\right) \leqq c_{\varepsilon}(\omega)+\varepsilon\left\|\varphi_{\varepsilon}\right\|_{L^{\alpha_{2}}}^{\alpha_{2}} \underset{\varepsilon \rightarrow \infty}{\rightarrow} c(\omega)
$$

Applying Lemma 5.1 to $\left(\varphi_{1 / n}\right)_{n \geqq 0}$, we find $\phi \in E$ such that $\left(I^{\omega}\right)^{\prime}(\phi)=0$, $0<\lambda_{0}<I^{\omega}(\phi) \leqq c(\omega)$.

From Proposition 3.2, $\phi$ is in $\bigcap_{q \geqq 2} W^{1, q}\left(\mathbb{R}^{3}, \mathbb{C}^{4}\right)$, and Theorem 3 is proved.

Acknowledgement. The second author thanks I. Ekeland for bringing non-linear Dirac theory to his attention. The authors thank P.L. Lions for stimulating discussions.

\section{References}

1. Ambrosetti, A., Rabinowitz, P.H.: Dual variational methods in critical points theory and applications. J. Funct. Anal. 14, 349-38 (1973)

2. Berestycki, H., Lions, P.-L.: Nonlinear scalar field equations. Arch. Rat. Mech. Anal. 82, 313-346 (1983)

3. Benci, V., Capozzi, A., Fortunato, D.: Periodic solutions of Hamiltonian systems with superquadratic potential. Ann. Mat. Pura et app. (IV), Vol. CXLIII, 1-46 (1986)

4. Bjorken, J.D., Drell, S.D.: Relativistic quantum fields. New York: McGraw-Hill, 1965

5. Balabane, M., Cazenave, T., Douady, A., Merle, F.: Existence of excited states for a nonlinear Dirac field. Commun. Math. Phys. 119, 153-176 (1988)

6. Balabane, M., Cazenave, T., Vazquez, L.: Existence of standing waves for Dirac fields with singular nonlinearities. Commun. Math. Phys. 133, 53-74 (1990)

7. Benci, V., Rabinowitz, P.H.: Critical point theorems for indefinite functionals, Inv. Math. 52, 336-352 (1979)

8. Cazenave, T.: On the existence of stationary states for classical non-linear Dirac fields. In: Hyperbolic systems and Mathematical Physics. Textos e Notas 4, CMAF, Lisbonne, 1989

9. Cazenave, T., Vazquez, L.: Existence of localized solutions for a classical nonlinear Dirac field. Commun. Math. Phys. 105, 35-47 (1986)

10. Finkelstein, R., Lelevier, R., Ruderman, M.: Phys. Rev. 83, 326-332 (1951)

11. Hofer, H., Wysocki, K.: First order elliptic systems and the existence of homoclinic orbits in Hamiltonian systems. Math. Ann. 288, 483-503 (1990)

12. Lions, P.-L.: The concentration-compactness method in the Calculus of Variations. The locally compact case. Part. I: Anal. non-linéaire, Ann. IHP 1, 109-145 (1984); Part. II: Anal. nonlinéaire, Ann. IHP 1, 223-283 (1984)

13. Merle, F.: Existence of stationary states for nonlinear Dirac equations. J. Diff. Eq. 74 (1), $50-68$ (1988)

14. Rañada, A.F.: Classical nonlinear Dirac field models of extended particles. In: Quantum theory, groups, fields and particles (editor A.O. Barut). Amsterdam, Reidel: 1982

15. Séré, E.: Homoclinic orbits on compact hypersurfaces in $\mathbb{R}^{2 N}$, of restricted contact type. Preprint CEREMADE 9238 (1992)

16. Smale, S.: An infinite dimensional version of Sard's theorem. Amer. J. Math. 87, 861-866 (1965)

17. Soler, M.: Phys. Rev. D1, 2766-2769 (1970)

18. Strauss, W.A.: Existence of solitary waves in higher dimensions. Commun. Math. Phys. 55, 149-162 (1977)

19. Tanaka, K.: Homoclinic orbits in the first order superquadratic Hamiltonian system: convergence of subharmonics. J. Diff. Eq. 94, 315-339 (1991) 